\title{
PAIRINGS BETWEEN BOUNDED DIVERGENCE-MEASURE VECTOR FIELDS AND BV FUNCTIONS
}

\author{
GRAZIANO CRASTA, VIRGINIA DE CICCO, AND ANNALISA MALUSA
}

\begin{abstract}
We introduce a family of pairings between a bounded divergence-measure vector field and a function $u$ of bounded variation, depending on the choice of the pointwise representative of $u$. We prove that these pairings inherit from the standard one, introduced in 6] 10, all the main properties and features (e.g. coarea, Leibniz and GaussGreen formulas). We also characterize the pairings making the corresponding functionals semicontinuous with respect to the strict convergence in $B V$. We remark that the standard pairing in general does not share this property.
\end{abstract}

\section{Contents}

1. Introduction

2. Notation and preliminary results

2.1. Measures

2.2. Functions of bounded variation

2.3. Divergence-measure fields

2.4. Weak normal traces

3. Some remarks on $L^{1}(\Omega,|\operatorname{div} \boldsymbol{A}|)$

4. Definition and basic properties of pairings

5. Coarea formula for generalized pairings

6. Chain rule, Leibniz and Gauss-Green formulas for generalized pairings

7. Semicontinuity results

References

\section{INTRODUCTION}

In the seminal papers $[6,10$, the product rule

$$
\operatorname{div}(u \boldsymbol{A})=u \operatorname{div} \boldsymbol{A}+\boldsymbol{A} \cdot \nabla u,
$$

for smooth functions $u$ and regular vector fields $\boldsymbol{A}$ in $\mathbb{R}^{N}$, has been suitably extended to $B V$ functions and bounded divergence-measure vector fields. In particular, Chen and Frid [10 showed, using a regularization argument, that there exists a finite Radon measure $(\boldsymbol{A}, D u)_{*}$, which coincides to $\boldsymbol{A} \cdot \nabla u \mathcal{L}^{N}$ in the smooth case, such that the relation

$$
\operatorname{div}(u \boldsymbol{A})=u^{*} \operatorname{div} \boldsymbol{A}+(\boldsymbol{A}, D u)_{*}
$$

Date: October 14, 2019.

2010 Mathematics Subject Classification. 26B30,49Q15,49J45.

Key words and phrases. Divergence-measure vector fields, functions of bounded variation, coarea formula, Gauss-Green formula, semicontinuity. 
holds in the sense of measures. The measure $(\boldsymbol{A}, D u)_{*}$, usually called Anzellotti's pairing and that we call in the sequel the standard pairing between $\boldsymbol{A}$ and $D u$, is then defined in terms of the precise representative $u^{*}$ of $u$, which is the pointwise value of $u$ obtained as limit of regularizations by convolutions.

The standard pairing turns out to be a basic tool in many applications. We mention here, among others: extensions of the Gauss-Green formula [6, 8, 9, 13, 17, 19, 31]; the setting of the Euler-Lagrange equations associated with integral functionals defined in $B V$ [4, 32, 33]; Dirichlet problems for equations involving the 1-Laplace operator [5, 8, 21, 22, 26, 27]; conservation laws [10 14, 18]; the Prescribed Mean Curvature problem and capillarity [30, 31]; continuum mechanics [9, 23, 37, 38].

On the other hand, the standard pairing is not adequate when dealing with obstacle problems in $B V$ (see [34 36]) or with semicontinuity properties, as we will explain below. The aim of this paper is to introduce a new family of pairings, depending on the choice of the pointwise representative of $u$, suitable to treat this kind of problems.

The main ingredients to build this family of pairings are the absolute continuity of the measure $\operatorname{div} \boldsymbol{A}$ with respect to the $(N-1)$-dimensional Hausdorff measure $\mathcal{H}^{N-1}$, and the fact that the pointwise value of a $B V$ function can be specified up to a $\mathcal{H}^{N-1}$-negligible set. Indeed, a $B V$ function $u$ is approximately continuous outside a singular set $S_{u}$ and its approximate upper and lower limits $u^{+}$and $u^{-}$coincide with the traces of $u$ on the countably $\mathcal{H}^{N-1}$-rectifiable jump set $J_{u} \subset S_{u}$, with $\mathcal{H}^{N-1}\left(S_{u} \backslash J_{u}\right)=0$ (see Section 2.2). Hence, a representative of $u$ can be defined by its approximate limit $\tilde{u}$ outside $S_{u}$ and through its traces $u^{ \pm}$on $J_{u}$. We remark again that the presence of $u^{*}:=\left(u^{+}+u^{-}\right) / 2$ in (21) as the pointwise representative of $u$ is due to the regularization argument used in [10] in order to define the standard pairing.

Recently, Scheven and Schmidt [34 36] have been in need to introduce the pairing

$$
(\boldsymbol{A}, D u)_{1}:=-u^{+} \operatorname{div} \boldsymbol{A}+\operatorname{div}(u \boldsymbol{A})
$$

in order to study weakly 1-superharmonic functions and minimization problems for the total variation with an obstacle. Indeed, in this case, the presence of the representative $u^{+}$ comes out from (11) using the one-sided approximation procedure of $u$ introduced in [7].

In this paper we prove that, for every Borel function $\lambda: \mathbb{R}^{N} \rightarrow[0,1]$, there exists a measure $(\boldsymbol{A}, D u)_{\lambda}$ such that

$$
\operatorname{div}(u \boldsymbol{A})=u^{\lambda} \operatorname{div} \boldsymbol{A}+(\boldsymbol{A}, D u)_{\lambda},
$$

where $u^{\lambda}:=(1-\lambda) u^{-}+\lambda u^{+}$is a selection of the multifunction $x \mapsto\left[u^{-}(x), u^{+}(x)\right]$. We show that, if the jump part $\operatorname{div}^{j} \boldsymbol{A}$ of $\operatorname{div} \boldsymbol{A}$ vanishes (see Proposition 2.3 for the definition), then $(\boldsymbol{A}, D u)_{\lambda}$ is independent of $\lambda$.

We show that this freedom in the choice of $u^{\lambda}$ is necessary in order to obtain semicontinuity results in $B V$ for the functionals

$$
F_{\varphi}(u):=\left\langle(\boldsymbol{A}, D u)_{\lambda}, \varphi\right\rangle, \quad \varphi \in C_{c}\left(\mathbb{R}^{N}\right), \quad \varphi \geq 0 .
$$

We characterize the selections $\lambda$ such that these functionals are lower (resp. upper) semicontinuous with respect to the strict convergence in $B V$. More precisely, denoting by $(\operatorname{div} \boldsymbol{A})^{ \pm}$the positive and the negative part of the measure $\operatorname{div} \boldsymbol{A}$, the choices of $\lambda$ which guarantee the lower semicontinuity of the functionals in (5) satisfy

$$
(\boldsymbol{A}, D u)_{\lambda}=-u^{+}(\operatorname{div} \boldsymbol{A})^{+}+u^{-}(\operatorname{div} \boldsymbol{A})^{-}+\operatorname{div}(u \boldsymbol{A}),
$$


whereas the upper semicontinuity is characterized by

$$
(\boldsymbol{A}, D u)_{\lambda}=-u^{-}(\operatorname{div} \boldsymbol{A})^{+}+u^{+}(\operatorname{div} \boldsymbol{A})^{-}+\operatorname{div}(u \boldsymbol{A}) .
$$

As a consequence, it is a matter of fact that, in general, the standard pairing does not share these semicontinuity properties. On the other hand, if div $\boldsymbol{A} \leq 0$, as in 34 36], from the above result follows that the pairing (3) is upper semicontinuous with respect to the strict convergence in $B V$.

The plan of the paper is the following. In Section 2 we recall some known results on $B V$ functions, divergence-measure vector fields and their weak normal traces. In Section 3 we focus our attention on the summability of $u^{\lambda}$ with respect to the measure $|\operatorname{div} \boldsymbol{A}|$ and on some related properties of the truncated functions. In Sections 4, 5 and 6 we introduce the generalized pairing and we prove that it inherits from the standard one all the main properties and features. More precisely, $(\boldsymbol{A}, D u)_{\lambda}$ is a Radon measure, absolutely continuous with respect to $|D u|$, it satisfies the coarea, the chain rule and the Leibniz formulas, and it is consistent with the Gauss-Green formula.

The proofs of these results are based on the analogous properties valid for the standard pairing (see [19]), the fact that the generalized pairing differs from the standard one only by a term concentrated on $J_{u}$ (see (20) ), and some representation results of the normal traces of $\boldsymbol{A}$ on $J_{u}$ (see [1]).

Our main application of the above theory is proposed in Section 7, where we consider the semicontinuity properties of the functionals $F_{\varphi}$ defined in (15), with respect to the strict convergence in $B V$. In Theorem 7.6 we prove the characterizations (6)-(7) of the semicontinuous pairings. The proof is based on a recent result of Lahti (see [28]), which assures the lower (upper) semicontinuity of the lower $u^{-}$(upper $u^{+}$) limit under the strict convergence in $B V$, combined with the one-sided approximation result in [7, and a very careful treatment of the jump part of the measure $\operatorname{div} \boldsymbol{A}$. We show by easy examples that no semicontinuity property has to be expected with respect to the weak* convergence in $B V$.

\section{Notation AND PRELIMINARY RESUlts}

In the following $\Omega$ will always denote a nonempty open subset of $\mathbb{R}^{N}$. For every $E \subset \Omega$, $\chi_{E}$ denotes its characteristic function. We say that $E_{h}$ converges to $E$ if $\chi_{E_{h}}$ converges to $\chi_{E}$ in $L^{1}(\Omega)$.

We denote by $\mathcal{L}^{N}$ and $\mathcal{H}^{N-1}$ the Lebesgue measure and the $(N-1)$-dimensional Hausdorff measure in $\mathbb{R}^{N}$, respectively.

If $E \subset \mathbb{R}^{N}$ is an open set, the notation $\varphi \nearrow \chi_{E}$ denotes any family $\left(\varphi_{j}\right)$ of smooth functions with support in $E$, such that $0 \leq \varphi_{j} \leq 1$, and $\lim _{j} \varphi_{j}(x)=1$ for every $x \in E$.

Given an $\mathcal{L}^{N}$-measurable set $E \subset \mathbb{R}^{N}$, For every $t \in[0,1]$ we denote by $E^{t}$ the set

$$
E^{t}:=\left\{x \in \mathbb{R}^{N}: \lim _{\rho \rightarrow 0^{+}} \frac{\mathcal{L}^{N}\left(E \cap B_{\rho}(x)\right)}{\mathcal{L}^{N}\left(B_{\rho}(x)\right)}=t\right\}
$$

of all points where $E$ has density $t$. The sets $E^{0}, E^{1}, \partial^{e} E:=\mathbb{R}^{N} \backslash\left(E^{0} \cup E^{1}\right)$ are called respectively the measure theoretic exterior, the measure theoretic interior and the essential boundary of $E$. 
Let $u: \Omega \rightarrow \mathbb{R}$ be a Borel function. We denote by $u^{-}$and $u^{+}$the approximate lower limit and the approximate upper limit of $u$, defined respectively by

$$
\begin{aligned}
& u^{+}(x):=\inf \{t \in \mathbb{R}:\{u>t\} \text { has density } 0 \text { at } x\}, \\
& u^{-}(x):=\sup \{t \in \mathbb{R}:\{u>t\} \text { has density } 1 \text { at } x\} .
\end{aligned}
$$

The function $u$ is approximately continuous at $x \in \Omega$ if $u^{+}(x)=u^{-}(x)$ and, in this case, we denote by $\widetilde{u}(x)$ the common value.

Given $u \in L_{\text {loc }}^{1}(\Omega), x \in \Omega$ is a Lebesgue point of $u$ (with respect to $\mathcal{L}^{N}$ ) if there exists $z \in \mathbb{R}$ such that

$$
\lim _{r \rightarrow 0^{+}} \frac{1}{\mathcal{L}^{N}\left(B_{r}(x)\right)} \int_{B_{r}(x)}|u(y)-z| d y=0 .
$$

In this case, $x$ is a point of approximate continuity, and $z=\widetilde{u}(x)$ (see 24, Proposition 1.163]). We denote by $S_{u} \subset \Omega$ the set of points where this property does not hold.

We say that $x \in \Omega$ is an approximate jump point of $u$ if there exist $a, b \in \mathbb{R}$ and a unit vector $\nu \in \mathbb{R}^{n}$ such that $a \neq b$ and

$$
\begin{aligned}
& \lim _{r \rightarrow 0^{+}} \frac{1}{\mathcal{L}^{N}\left(B_{r}^{i}(x)\right)} \int_{B_{r}^{i}(x)}|u(y)-a| d y=0, \\
& \lim _{r \rightarrow 0^{+}} \frac{1}{\mathcal{L}^{N}\left(B_{r}^{e}(x)\right)} \int_{B_{r}^{e}(x)}|u(y)-b| d y=0,
\end{aligned}
$$

where $B_{r}^{i}(x):=\left\{y \in B_{r}(x):(y-x) \cdot \nu>0\right\}$, and $B_{r}^{e}(x):=\left\{y \in B_{r}(x):(y-x)\right.$. $\nu<0\}$. The triplet $(a, b, \nu)$, uniquely determined by (8) up to a permutation of $(a, b)$ and a simultaneous change of sign of $\nu$, is denoted by $\left(u^{i}(x), u^{e}(x), \nu_{u}(x)\right)$. The set of approximate jump points of $u$ will be denoted by $J_{u}$.

A $\mathcal{H}^{N-1}$-measurable set $E \subset \mathbb{R}^{N}$ is countably $\mathcal{H}^{N-1}$-rectifiable if there exist countably many $C^{1}$ graphs $\left(\Sigma_{i}\right)_{i \in \mathbb{N}}$ such that $\mathcal{H}^{N-1}\left(E \backslash \bigcup_{i} \Sigma_{i}\right)=0$.

2.1. Measures. The space of all Radon measures on $\Omega$ will be denoted by $\mathcal{M}(\Omega)$.

Given $\mu \in \mathcal{M}(\Omega)$, its total variation $|\mu|$ is the nonnegative Radon measure defined by

$$
|\mu|(E):=\sup \left\{\sum_{h=0}^{\infty}\left|\mu\left(E_{h}\right)\right|: E_{h} \mu \text {-measurable sets, pairwise disjoint, } E=\bigcup_{h=0}^{\infty} E_{h}\right\},
$$

for every $\mu$-measurable set $E$ and its positive and negative parts are defined, respectively, by

$$
\mu^{+}:=\frac{|\mu|+\mu}{2}, \quad \mu^{-}:=\frac{|\mu|-\mu}{2} .
$$

If $\mu_{1}, \mu_{2} \in \mathcal{M}(\Omega)$, then $\max \left\{\mu_{1}, \mu_{2}\right\}$ (resp. $\min \left\{\mu_{1}, \mu_{2}\right\}$ ) is the measure that assigns to every Borel set $E \subset \Omega$, the supremum (resp. infimum) of $\mu_{1}\left(E_{1}\right)+\mu_{2}\left(E_{2}\right)$ among all pairwise disjoint Borel sets $E_{1}, E_{2}$ such that $E_{1} \cup E_{2}=E$.

Given $\mu \in \mathcal{M}(\Omega)$ and a $\mu$-measurable set $E$, the restriction $\mu\llcorner E$ is the Radon measure defined by

$$
\mu\llcorner E(B)=\mu(E \cap B), \quad \forall B \mu \text {-measurable, } B \subset \Omega .
$$

We recall the following property (see [3], Proposition 2.56 and formula (2.41)):

$$
E \subset \Omega,|\mu|(E)=0 \Longrightarrow|\mu|\left(B_{r}(x)\right)=o\left(r^{N-1}\right) \text { for } \mathcal{H}^{N-1} \text {-a.e. } x \in E .
$$

Given a nonnegative Borel measure $\nu$, we say that $\mu \in \mathcal{M}(\Omega)$ is absolutely continuous with respect to $\nu$ (and we write $\mu \ll \nu$ ), if $|\mu|(B)=0$ for every set $B$ such that $\nu(B)=0$. 
We say that two positive measures $\nu_{1}, \nu_{2} \in \mathcal{M}(\Omega)$ are mutually singular (and we write $\left.\nu_{1} \perp \nu_{2}\right)$ if there exists a Borel set $E$ such that $\left|\nu_{1}\right|(E)=0$ and $\left|\nu_{2}\right|(\Omega \backslash E)=0$.

By the Radon-Nikodým theorem, given a nonnegative Radon measure $\nu$, every $\mu \in$ $\mathcal{M}(\Omega)$ can be uniquely decomposed as $\mu=\mu_{1}+\mu_{2}$ with $\mu_{1} \ll \nu$ and $\mu_{2} \perp \nu$, and there exists a unique function (called the density of $\mu$ with respect to $\nu) \psi_{\nu} \in L^{1}(\Omega, \nu)$ such that $\mu_{1}=\psi_{\nu} \nu$. In particular, since $\mu \ll|\mu|$, then there exists $\psi \in L^{1}(\Omega,|\mu|)$, with $|\psi|=1$ $|\mu|-$ a.e. in $\Omega$, and such that $\mu=\psi|\mu|$. This is usually called the polar decomposition of $\mu$.

The following lemma shows the relation between the densities of $\mu$ and $|\mu|$, where $\mu$ is a Radon measure absolutely continuous with respect to $\mathcal{H}^{N-1}$.

Lemma 2.1. Let $\mu \ll \mathcal{H}^{N-1}$ be a Radon measure in $\Omega$, and let $\mu=\psi|\mu|$ be its polar decomposition. Then there exists a Borel set $Z \subset \Omega$, with $|\mu|(Z)=0$, such that every $x \in \Omega \backslash Z$ is a Lebesgue point of $\psi$ with respect to $|\mu|$, and

$$
\exists \lim _{r \searrow 0} \frac{|\mu|\left(B_{r}(x)\right)}{r^{N-1}}=L \in \mathbb{R} \quad \Longleftrightarrow \quad \exists \lim _{r \searrow 0} \frac{\mu\left(B_{r}(x)\right)}{r^{N-1}}=\psi(x) L .
$$

Proof. Let $A \subset \Omega$ be the set of Lebesgue points of $\psi$ with respect to $|\mu|$. By [3, Corollary 2.23], we have that $|\mu|(\Omega \backslash A)=0$. Since $|\psi|=1|\mu|$-a.e., it is not restrictive to assume that

$$
|\psi(x)|=1, \quad \lim _{r \searrow 0} \frac{1}{|\mu|\left(B_{r}(x)\right)} \int_{B_{r}(x)}|\psi(y)-\psi(x)| d|\mu|=0, \quad \forall x \in A .
$$

Moreover, from [3, Theorem 2.56 and (2.40)], the set

$$
Z_{1}:=\left\{x \in \Omega: \limsup _{r \searrow 0} \frac{|\mu|\left(B_{r}(x)\right)}{r^{N-1}}=+\infty\right\}
$$

has zero $\mathcal{H}^{N-1}$-measure, hence also $|\mu|\left(Z_{1}\right)=0$.

If we set $Z:=(\Omega \backslash A) \cup Z_{1}$, then $|\mu|(Z)=0$ and (10) holds in $\Omega \backslash Z$. Specifically, given $x \in \Omega \backslash Z, B_{r}(x) \subset \Omega$ and $\varphi \in C_{c}(\Omega)$ with support in $B_{r}(x)$, since $|\psi(x)|=1$, we have that $|1-\psi(y) \psi(x)|=|\psi(y)-\psi(x)|$, and hence

$$
\begin{aligned}
\left|\int_{\Omega} \varphi d\right| \mu\left|-\psi(x) \int_{\Omega} \varphi d \mu\right| & =\left|\int_{B_{r}(x)} \varphi(y)[1-\psi(y) \psi(x)] d\right| \mu|(y)| \\
& \leq\|\varphi\|_{\infty}|\mu|\left(B_{r}(x)\right) f_{B_{r}(x)}|\psi(y)-\psi(x)| d|\mu|(y) .
\end{aligned}
$$

Taking $\varphi \nearrow \chi_{B_{r}(x)}$ and dividing by $r^{N-1}$ we finally get

$$
\left|\frac{|\mu|\left(B_{r}(x)\right)}{r^{N-1}}-\psi(x) \frac{\mu\left(B_{r}(x)\right)}{r^{N-1}}\right| \leq \frac{|\mu|\left(B_{r}(x)\right)}{r^{N-1}} f_{B_{r}(x)}|\psi(y)-\psi(x)| d|\mu|(y),
$$

hence (10) follows because $x \notin Z_{1}$ and $x$ is a Lebesgue point of $\psi$.

Given $\mu \in \mathcal{M}(\Omega)$, we denote by $\mu=\mu^{a}+\mu^{s}$ its Lebesgue decomposition in the absolutely continuous part $\mu^{a} \ll \mathcal{L}^{N}$ and the singular part $\mu^{s} \perp \mathcal{L}^{N}$. We recall a relevant decomposition result for $\mu^{s}$ (see [2], Proposition 5).

Proposition 2.2. If $\mu \in \mathcal{M}(\Omega)$ is such that $\mu^{s} \ll \mathcal{H}^{N-1}$, then $\mu^{s}$ can be uniquely decomposed as the sum $\mu^{j}+\mu^{c}$, where $\mu^{j}, \mu^{c} \in \mathcal{M}(\Omega)$ are two mutually singular measures having the following properties:

(i) $\mu^{c}(B)=0$ for every $B$ such that $\mathcal{H}^{N-1}(B)<+\infty$; 
(ii) the set

$$
\Theta_{\mu}:=\left\{x \in \Omega: \limsup _{r \rightarrow 0+} \frac{|\mu|\left(B_{r}(x)\right)}{r^{N-1}}>0\right\}
$$

is a Borel set, $\sigma$-finite with respect to $\mathcal{H}^{N-1}$;

(iii) there exists $f \in L^{1}\left(\Theta_{\mu}, \mathcal{H}^{N-1}\left\llcorner\Theta_{\mu}\right)\right.$ such that $\mu^{j}=f \mathcal{H}^{N-1}\left\llcorner\Theta_{\mu}\right.$.

The measures $\mu^{j}, \mu^{c}$ are called jump part and Cantor part of the measure $\mu$, while $\Theta_{\mu}$ is called jump set of $\mu$.

2.2. Functions of bounded variation. We say that $u \in L^{1}(\Omega)$ is a function of bounded variation in $\Omega$ if the distributional derivative $D u$ of $u$ is a finite Radon measure in $\Omega$. The vector space of all functions of bounded variation in $\Omega$ will be denoted by $B V(\Omega)$. Moreover, we will denote by $B V_{\text {loc }}(\Omega)$ the set of functions $u \in L_{\text {loc }}^{1}(\Omega)$ that belongs to $B V(A)$ for every open set $A \Subset \Omega$ (i.e., the closure $\bar{A}$ of $A$ is a compact subset of $\Omega$ ).

If $u \in B V(\Omega)$, then $D u$ can be decomposed as the sum of the absolutely continuous and the singular part with respect to the Lebesgue measure, i.e.

$$
D u=D^{a} u+D^{s} u, \quad D^{a} u=\nabla u \mathcal{L}^{N},
$$

where $\nabla u$ is the approximate gradient of $u$, defined $\mathcal{L}^{N}$-a.e. in $\Omega$ (see [3, Section 3.9]). The jump set $J_{u}$ has the following properties: it is countably $\mathcal{H}^{N-1}$-rectifiable and $\mathcal{H}^{N-1}\left(S_{u}\right)$ $\left.J_{u}\right)=0$ (see [3. Definition 2.57 and Theorem 3.78]); it is contained in the set $\Theta_{D u}$ defined in Proposition 2.2(ii) with $\mu=D u$, and $\mathcal{H}^{N-1}\left(\Theta_{D u} \backslash J_{u}\right)=0$ (see [3. Proposition 3.92(b)]). By Proposition 2.2, the singular part $D^{s} u$ can be further decomposed as the sum of its Cantor and jump part, i.e. $D^{s} u=D^{c} u+D^{j} u, D^{c} u:=D^{s} u\left\llcorner\left(\Omega \backslash S_{u}\right)\right.$, and

$$
D^{j} u:=D^{s} u\left\llcorner J_{u}=\left(u^{i}-u^{e}\right) \nu_{u} \mathcal{H}^{N-1}\left\llcorner J_{u} .\right.\right.
$$

We denote by $D^{d} u:=D^{a} u+D^{c} u$ the diffuse part of the measure $D u$.

At every point $x \in J_{u}$ we have that $-\infty<u^{-}(x)<u^{+}(x)<+\infty$ and

$$
u^{-}(x)=\min \left\{u^{i}(x), u^{e}(x)\right\}, \quad u^{+}(x)=\max \left\{u^{i}(x), u^{e}(x)\right\}, \quad x \in J_{u} .
$$

Moreover, we can always choose an orientation on $J_{u}$ such that $u^{i}=u^{+}$on $J_{u}$ (see [25, 44.1 .4 , Theorem 2]). In the following we shall always extend the functions $u^{i}, u^{e}$ to $\Omega \backslash\left(S_{u} \backslash J_{u}\right)$ by setting

$$
u^{i}=u^{e}=\widetilde{u} \quad \text { in } \Omega \backslash S_{u} .
$$

Given a Borel function $\lambda: \Omega \rightarrow[0,1]$, the $\lambda$-representative of $u \in B V_{\text {loc }}(\Omega)$ is defined by

$$
u^{\lambda}(x):= \begin{cases}\tilde{u}(x), & x \in \Omega \backslash S_{u}, \\ (1-\lambda(x)) u^{-}(x)+\lambda(x) u^{+}(x), & x \in J_{u} .\end{cases}
$$

When $\lambda(x)=1 / 2$ for every $x \in \Omega$, the $\lambda$-representative coincides with the precise representative $u^{*}:=\left(u^{+}+u^{-}\right) / 2$ of $u$.

Let $E$ be an $\mathcal{L}^{N}$-measurable subset of $\mathbb{R}^{N}$. For every open set $\Omega \subset \mathbb{R}^{N}$ the perimeter $P(E, \Omega)$ is defined by

$$
P(E, \Omega):=\sup \left\{\int_{E} \operatorname{div} \varphi d x: \varphi \in C_{c}^{1}\left(\Omega, \mathbb{R}^{N}\right),\|\varphi\|_{\infty} \leq 1\right\} .
$$

We say that $E$ is of finite perimeter in $\Omega$ if $P(E, \Omega)<+\infty$.

Denoting by $\chi_{E}$ the characteristic function of $E$, if $E$ is a set of finite perimeter in $\Omega$, then $D \chi_{E}$ is a finite Radon measure in $\Omega$ and $P(E, \Omega)=\left|D \chi_{E}\right|(\Omega)$. 
If $\Omega \subset \mathbb{R}^{N}$ is the largest open set such that $E$ is locally of finite perimeter in $\Omega$, we call reduced boundary $\partial^{*} E$ of $E$ the set of all points $x \in \Omega$ in the support of $\left|D \chi_{E}\right|$ such that the limit

$$
\widetilde{\nu}_{E}(x):=\lim _{\rho \rightarrow 0^{+}} \frac{D \chi_{E}\left(B_{\rho}(x)\right)}{\left|D \chi_{E}\right|\left(B_{\rho}(x)\right)}
$$

exists in $\mathbb{R}^{N}$ and satisfies $\left|\widetilde{\nu}_{E}(x)\right|=1$. The function $\widetilde{\nu}_{E}: \partial^{*} E \rightarrow S^{N-1}$ is called the measure theoretic unit interior normal to $E$.

A fundamental result of De Giorgi (see [3, Theorem 3.59]) states that $\partial^{*} E$ is countably $(N-1)$-rectifiable and $\left|D \chi_{E}\right|=\mathcal{H}^{N-1} \mathbf{L} \partial^{*} E$. If $E$ has finite perimeter in $\Omega$, Federer's structure theorem states that $\partial^{*} E \cap \Omega \subset E^{1 / 2} \subset \partial^{e} E$ and $\mathcal{H}^{N-1}\left(\Omega \backslash\left(E^{0} \cup \partial^{e} E \cup E^{1}\right)\right)=0$ (see [3, Theorem 3.61]).

2.3. Divergence-measure fields. We will denote by $\mathcal{D} \mathcal{M}^{\infty}(\Omega)$ the space of all vector fields $\boldsymbol{A} \in L^{\infty}\left(\Omega, \mathbb{R}^{N}\right)$ whose divergence in the sense of distributions is a finite Radon measure in $\Omega$, acting as

$$
\int_{\Omega} \varphi d \operatorname{div} \boldsymbol{A}=-\int_{\Omega} \boldsymbol{A} \cdot \nabla \varphi d x \quad \forall \varphi \in C_{c}^{\infty}(\Omega) .
$$

Similarly, $\mathcal{D M}_{\mathrm{loc}}^{\infty}(\Omega)$ will denote the space of all vector fields $\boldsymbol{A} \in L_{\text {loc }}^{\infty}\left(\Omega, \mathbb{R}^{N}\right)$ whose divergence in the sense of distributions is a Radon measure in $\Omega$.

The basic properties of these vector fields are collected in the following proposition.

Proposition 2.3. Let $\boldsymbol{A}$ be a vector field belonging to $\mathcal{D} \mathcal{M}^{\infty}(\Omega)$, and let $\Theta_{\boldsymbol{A}}$ be the jump set of the measure $\mu=|\operatorname{div} \boldsymbol{A}|$, defined in Proposition 2.2(ii). Then the following hold.

(i) $|\operatorname{div} \boldsymbol{A}| \ll \mathcal{H}^{N-1}$;

(ii) $\Theta_{\boldsymbol{A}}$ is a Borel set, $\sigma$-finite with respect to $\mathcal{H}^{N-1}$;

(iii) $\operatorname{div} \boldsymbol{A}=\operatorname{div}^{a} \boldsymbol{A}+\operatorname{div}^{c} \boldsymbol{A}+\operatorname{div}^{j} \boldsymbol{A}$, where $\operatorname{div}^{a} \boldsymbol{A}$ is absolutely continuous with respect to $\mathcal{L}^{N}$, $\operatorname{div}^{c} \boldsymbol{A}(B)=0$ for every set $B$ with $\mathcal{H}^{N-1}(B)<+\infty$, and there exists $f \in L^{1}\left(\Theta_{\boldsymbol{A}}, \mathcal{H}^{N-1}\left\llcorner\Theta_{\boldsymbol{A}}\right)\right.$ such that $\operatorname{div}^{j} \boldsymbol{A}=f \mathcal{H}^{N-1}\left\llcorner\Theta_{\boldsymbol{A}}\right.$.

Proof. The main property (i) is proved in [10, Proposition 3.1]. The decomposition then follows from Proposition 2.2 .

2.4. Weak normal traces. In what follows, we will deal with the traces of the normal component of a vector field $\boldsymbol{A} \in \mathcal{D M}^{\infty}(\Omega)$ on a countably $\mathcal{H}^{N-1}$-rectifiable set $\Sigma \subset \Omega$. In order to fix the notation, we briefly recall the construction given in [1] (see Propositions 3.2, 3.4 and Definition 3.3).

Given a domain $\Omega^{\prime} \Subset \Omega$ of class $C^{1}$, the trace of the normal component of $\boldsymbol{A}$ on $\partial \Omega^{\prime}$ is the distribution defined by

$$
\left\langle\operatorname{Tr}\left(\boldsymbol{A}, \partial \Omega^{\prime}\right), \varphi\right\rangle:=\int_{\Omega^{\prime}} \boldsymbol{A} \cdot \nabla \varphi d x+\int_{\Omega^{\prime}} \varphi d \operatorname{div} \boldsymbol{A}, \quad \forall \varphi \in C_{c}^{\infty}(\Omega) .
$$

It turns out that this distribution is induced by an $L^{\infty}$ function on $\partial \Omega^{\prime}$, still denoted by $\operatorname{Tr}\left(\boldsymbol{A}, \partial \Omega^{\prime}\right)$, and

$$
\left\|\operatorname{Tr}\left(\boldsymbol{A}, \partial \Omega^{\prime}\right)\right\|_{L^{\infty}\left(\partial \Omega^{\prime}, \mathcal{H}^{N-1}\left\llcorner\partial \Omega^{\prime}\right)\right.} \leq\|\boldsymbol{A}\|_{L^{\infty}\left(\Omega^{\prime}\right)} .
$$

Given a countably $\mathcal{H}^{N-1}$-rectifiable set $\Sigma$, there exist a covering $\left(\Sigma_{i}\right)_{i \in \mathbb{N}}$ of $\Sigma$ and Borel sets $N_{i} \subseteq \Sigma_{i}$ with the following properties:

(R1) $\Sigma_{i}$ is an oriented $C^{1}$ hypersurface, with (classical) normal vector field $\nu_{\Sigma_{i}}$;

(R2) $N_{i} \subseteq \Sigma_{i}$ are pairwise disjoint Borel sets such that $\mathcal{H}^{N-1}\left(\Sigma \backslash \bigcup_{i} N_{i}\right)=0$; 
(R3) for every $i \in \mathbb{N}$, there exist two open bounded sets $\Omega_{i}, \Omega_{i}^{\prime}$ with $C^{1}$ boundary and exterior normal vectors $\nu_{\Omega_{i}}$ and $\nu_{\Omega_{i}^{\prime}}$ respectively, such that $N_{i} \subseteq \partial \Omega_{i} \cap \partial \Omega_{i}^{\prime}$, and

$$
\nu_{\Sigma_{i}}(x)=\nu_{\Omega_{i}}(x)=-\nu_{\Omega_{i}^{\prime}}(x) \quad \forall x \in N_{i} .
$$

We can fix an orientation on $\Sigma$, given by

$$
\nu_{\Sigma}(x):=\nu_{\Sigma_{i}}(x), \quad \mathcal{H}^{N-1}-\text { a.e. on } N_{i}
$$

and the normal traces of $\boldsymbol{A}$ on $\Sigma$ are defined by

$$
\operatorname{Tr}^{e}(\boldsymbol{A}, \Sigma):=\operatorname{Tr}\left(\boldsymbol{A}, \partial \Omega_{i}\right), \quad \operatorname{Tr}^{i}(\boldsymbol{A}, \Sigma):=-\operatorname{Tr}\left(\boldsymbol{A}, \partial \Omega_{i}^{\prime}\right), \quad \mathcal{H}^{N-1}-\text { a.e. on } N_{i} .
$$

By a deep localization property proved in [1, Proposition 3.2], these definitions are independent of the choice of $\Sigma_{i}$ and $N_{i}$. In what follows, the pair $\left(\Sigma, \nu_{\Sigma}\right)$ (or, simply, $\Sigma$ ) will be called and oriented countably $\mathcal{H}^{N-1}$-rectifiable set.

We remark that, the normal traces belong to $L^{\infty}\left(\Sigma, \mathcal{H}^{N-1} \mathbf{L} \Sigma\right)$ and

$$
\operatorname{div} \boldsymbol{A} L \Sigma=\left[\operatorname{Tr}^{i}(\boldsymbol{A}, \Sigma)-\operatorname{Tr}^{e}(\boldsymbol{A}, \Sigma)\right] \mathcal{H}^{N-1}\llcorner\Sigma
$$

(see [1, Proposition 3.4]). In particular, by (13) $),|\operatorname{div} \boldsymbol{A}|(\Sigma) \leq\|\boldsymbol{A}\|_{\infty} \mathcal{H}^{N-1}(\Sigma)$.

Remark 2.4. We observe that, if $\Sigma$ is oriented by a normal vector field $\nu$ and $\Sigma^{\prime}$ is the same set oriented by $\nu^{\prime}:=-\nu$, then

$$
\operatorname{Tr}^{e}\left(\boldsymbol{A}, \Sigma^{\prime}\right)=-\operatorname{Tr}^{i}(\boldsymbol{A}, \Sigma), \quad \operatorname{Tr}^{i}\left(\boldsymbol{A}, \Sigma^{\prime}\right):=-\operatorname{Tr}^{e}(\boldsymbol{A}, \Sigma),
$$

so that the difference $\operatorname{Tr}^{i}(\boldsymbol{A}, \Sigma)-\operatorname{Tr}^{e}(\boldsymbol{A}, \Sigma)$ is independent of the choice of the orientation on $\Sigma$.

The following result is a consequence of (14) and will be used in the study of the semicontinuity of the generalized pairing (see Theorem [7.6).

Theorem 2.5. Let $\boldsymbol{A} \in \mathcal{D} \mathcal{M}^{\infty}(\Omega)$, let $\operatorname{div} \boldsymbol{A}=\psi_{\boldsymbol{A}}|\operatorname{div} \boldsymbol{A}|$ be the polar decomposition of the measure $\operatorname{div} \boldsymbol{A}$, and let $\Sigma \subset \Omega$ be an oriented countably $\mathcal{H}^{N-1}$-rectifiable set. Then

(16) $\operatorname{Tr}^{i}(\boldsymbol{A}, \Sigma)(x)-\operatorname{Tr}^{e}(\boldsymbol{A}, \Sigma)(x)=\psi_{\boldsymbol{A}}(x) \lim _{r \searrow 0} \frac{|\operatorname{div} \boldsymbol{A}|\left(B_{r}(x)\right)}{\omega_{N-1} r^{N-1}}, \quad$ for $\mathcal{H}^{N-1}$-a.e. $x \in \Sigma$.

Proof. From (9) with $\mu:=|\operatorname{div} \boldsymbol{A}|\llcorner\Sigma$ and $E:=\Omega \backslash \Sigma$, we have that

$$
\lim _{r \searrow 0} \frac{|\operatorname{div} \boldsymbol{A}|\left\llcorner\left(\mathbb{R}^{N} \backslash \Sigma\right)\left(B_{r}(x)\right)\right.}{\omega_{N-1} r^{N-1}}=0, \quad \text { for } \mathcal{H}^{N-1} \text {-a.e. } x \in \Sigma .
$$

On the other hand, by (14)

$$
\lim _{r \searrow 0} \frac{\operatorname{div} \boldsymbol{A} L \Sigma\left(B_{r}(x)\right)}{\omega_{N-1} r^{N-1}}=\operatorname{Tr}^{i}(\boldsymbol{A}, \Sigma)(x)-\operatorname{Tr}^{e}(\boldsymbol{A}, \Sigma)(x), \quad \text { for } \mathcal{H}^{N-1} \text {-a.e. } x \in \Sigma,
$$

and hence (15) holds.

Let us define the sets

$$
\begin{aligned}
& \Sigma^{\prime}:=\left\{x \in \Sigma: \quad \exists \lim _{r \searrow 0} \frac{|\operatorname{div} \boldsymbol{A}|\left(B_{r}(x)\right)}{\omega_{N-1} r^{N-1}}=0\right\}, \\
& \Sigma^{\prime \prime}:=\left\{x \in \Sigma: \quad \exists \lim _{r \searrow 0} \frac{|\operatorname{div} \boldsymbol{A}|\left(B_{r}(x)\right)}{\omega_{N-1} r^{N-1}}>0\right\} .
\end{aligned}
$$


By Proposition 2.3(i) and [3, Theorems 2.22 and 2.83], we infer that $\mathcal{H}^{N-1}\left(\Sigma \backslash\left(\Sigma^{\prime} \cup \Sigma^{\prime \prime}\right)\right)=$ 0 . From (15) and Lemma 2.1 we deduce that the equality in (16) holds for $\mathcal{H}^{N-1}$-a.e. $x \in \Sigma^{\prime \prime}$. On the other hand, from (15) we deduce that $\operatorname{Tr}^{i}(\boldsymbol{A}, \Sigma)(x)-\operatorname{Tr}^{e}(\boldsymbol{A}, \Sigma)(x)=0$ for $\mathcal{H}^{N-1}$-a.e. $x \in \Sigma^{\prime}$, hence (16) follows.

For later use, we recall here a result proved in [19, Proposition 3.1].

Proposition 2.6. Let $\boldsymbol{A} \in \mathcal{D M}^{\infty}(\Omega), u \in B V(\Omega) \cap L^{\infty}(\Omega)$ and let $\Sigma \subset \Omega$ be an oriented countably $\mathcal{H}^{N-1}$-rectifiable set. Then $u \boldsymbol{A} \in \mathcal{D} \mathcal{M}^{\infty}(\Omega)$ and the normal traces of $u \boldsymbol{A}$ on $\Sigma$ are given by

$$
\begin{aligned}
& \operatorname{Tr}^{e}(u \boldsymbol{A}, \Sigma)= \begin{cases}u^{e} \operatorname{Tr}^{e}(\boldsymbol{A}, \Sigma), & \mathcal{H}^{N-1}-\text { a.e. in } J_{u} \cap \Sigma, \\
\widetilde{u} \operatorname{Tr}^{e}(\boldsymbol{A}, \Sigma), & \mathcal{H}^{N-1}-\text { a.e. in } \Sigma \backslash J_{u} .\end{cases} \\
& \operatorname{Tr}^{i}(u \boldsymbol{A}, \Sigma)= \begin{cases}u^{i} \operatorname{Tr}^{i}(\boldsymbol{A}, \Sigma), & \mathcal{H}^{N-1}-\text { a.e. in } J_{u} \cap \Sigma, \\
\widetilde{u} \operatorname{Tr}^{i}(\boldsymbol{A}, \Sigma), & \mathcal{H}^{N-1}-\text { a.e. in } \Sigma \backslash J_{u} .\end{cases}
\end{aligned}
$$

\section{Some Remarks on $L^{1}(\Omega,|\operatorname{div} \boldsymbol{A}|)$}

In this section we analyze the properties of the functional spaces needed to define the pairing $(\boldsymbol{A}, D u)_{\lambda}$ introduced in (4).

Definition 3.1. Given $\boldsymbol{A} \in \mathcal{D} \mathcal{M}^{\infty}(\Omega)$, let us define the spaces:

$$
\begin{aligned}
B V(\Omega) \cap L^{1}(\Omega,|\operatorname{div} \boldsymbol{A}|) & :=\left\{u \in B V(\Omega): u^{*} \in L^{1}(\Omega,|\operatorname{div} \boldsymbol{A}|)\right\}, \\
B V_{\mathrm{loc}}(\Omega) \cap L_{\mathrm{loc}}^{1}(\Omega,|\operatorname{div} \boldsymbol{A}|) & :=\left\{u \in B V_{\mathrm{loc}}(\Omega): u^{*} \in L_{\mathrm{loc}}^{1}(\Omega,|\operatorname{div} \boldsymbol{A}|)\right\} .
\end{aligned}
$$

Notice that $|\operatorname{div} \boldsymbol{A}| \ll \mathcal{H}^{N-1}$ and $u^{*}$ is defined $\mathcal{H}^{N-1}$-a.e. in $\Omega$, hence the definitions are well-posed.

The following lemma shows that if $u \in B V(\Omega) \cap L^{1}(\Omega,|\operatorname{div} \boldsymbol{A}|)$ then any representative $u^{\lambda}$ of $u$ defined in (11) (in particular $u^{+}, u^{-}$) is summable with respect to the measure $|\operatorname{div} \boldsymbol{A}|$, hence the definitions of the spaces $B V(\Omega) \cap L^{1}(\Omega,|\operatorname{div} \boldsymbol{A}|)$ and $B V(\Omega) \cap$ $L^{1}(\Omega,|\operatorname{div} \boldsymbol{A}|)$ are independent of the choice of the pointwise representative.

Lemma 3.2. Let $\boldsymbol{A} \in \mathcal{D} \mathcal{M}^{\infty}(\Omega)$ and let $u \in B V_{\text {loc }}(\Omega)$. Given two Borel selections $\lambda, \mu: \Omega \rightarrow[0,1]$, then it holds:

(i) $u^{\lambda} \in L_{\mathrm{loc}}^{1}(\Omega,|\operatorname{div} \boldsymbol{A}|)$ if and only if $u^{\mu} \in L_{\mathrm{loc}}^{1}(\Omega,|\operatorname{div} \boldsymbol{A}|)$;

(ii) for every countably $\mathcal{H}^{N-1}$-rectifiable set $\Sigma \subset \Omega, u^{\lambda} \in L_{\mathrm{loc}}^{1}\left(\Sigma, \mathcal{H}^{N-1} \mathbf{L} \Sigma\right)$ if and only if $u^{\mu} \in L_{\text {loc }}^{1}\left(\Sigma, \mathcal{H}^{N-1}\llcorner\Sigma)\right.$.

Proof. We prove only (i), being the proof of (ii) entirely similar. By the representation (14) of $\operatorname{div} A\left\llcorner J_{u}\right.$ and the estimate (13), for every compact set $K \Subset \Omega$ we have

$$
\begin{aligned}
\int_{J_{u} \cap K}\left(u^{+}-u^{-}\right) d|\operatorname{div} \boldsymbol{A}| & =\int_{J_{u} \cap K}\left(u^{+}-u^{-}\right)\left|\operatorname{Tr}^{i}\left(\boldsymbol{A}, J_{u}\right)-\operatorname{Tr}^{e}\left(\boldsymbol{A}, J_{u}\right)\right| d \mathcal{H}^{N-1} \\
& \leq 2\|\boldsymbol{A}\|_{L^{\infty}(K)}\left|D^{j} u\right|(K) .
\end{aligned}
$$

Recalling that $u^{+}-u^{-}=0$ in $\Omega \backslash S_{u}$, i.e. $\mathcal{H}^{N-1}$-a.e. in $\Omega \backslash J_{u}$, it follows that $u^{+}-u^{-} \in$ $L_{\text {loc }}^{1}(\Omega,|\operatorname{div} \boldsymbol{A}|)$. The result now follows by observing that $u^{\lambda}=u^{\mu}+(\lambda-\mu)\left(u^{+}-u^{-}\right)$.

We underline that, for every $\boldsymbol{A} \in \mathcal{D M}^{\infty}(\Omega)$ and every $u \in B V(\Omega)$, it holds

$$
\int_{J_{u}}\left|\operatorname{Tr}^{i, e}\left(\boldsymbol{A}, J_{u}\right)\right|\left(u^{+}-u^{-}\right) d \mathcal{H}^{N-1} \leq\|\boldsymbol{A}\|_{\infty}\left|D^{j} u\right|(\Omega)<+\infty .
$$


Nevertheless, in general the functions $\left|\operatorname{Tr}^{i, e}\left(\boldsymbol{A}, J_{u}\right)\right| u^{ \pm}$are not summable with respect to $\mathcal{H}^{N-1}\left\llcorner J_{u}\right.$, even under the additional assumption $u \in B V(\Omega) \cap L^{1}(\Omega,|\operatorname{div} \boldsymbol{A}|)$, as it is shown in the following example.

Example 3.3. Let $\Omega=B_{1}(0) \subset \mathbb{R}^{2}$. Let us show that there exist a vector field $\boldsymbol{A} \in$ $\mathcal{D M}^{\infty}(\Omega)$ and a function $u \in B V(\Omega) \cap L^{1}(\Omega,|\operatorname{div} \boldsymbol{A}|)$ such that

$$
\int_{J_{u}}\left|\operatorname{Tr}^{i, e}\left(\boldsymbol{A}, J_{u}\right)\right| u^{ \pm} d \mathcal{H}^{1}=+\infty .
$$

Let $1=r_{0}>r_{1}>\cdots>r_{n}>\cdots$ be a decreasing sequence converging to 0 , such that

$$
\sum_{j} r_{j}<+\infty, \quad \sum_{j} j r_{j}=+\infty,
$$

and let $u: \Omega \rightarrow \mathbb{R}$ be defined by $u(x)=j$, if $r_{j} \leq|x|<r_{j-1}, j \in \mathbb{N}$. Since

$$
\int_{\Omega} u d x=\pi \sum_{j=1}^{\infty} r_{j}^{2}<\infty, \quad D u=\sum_{j=1}^{\infty} \mathcal{H}^{1}\left\llcorner\partial B_{r_{j}}(0), \quad|D u|(\Omega)=2 \pi \sum_{j=1}^{\infty} r_{j}<\infty,\right.
$$

then $u \in B V(\Omega)$. We choose on the jump set $J_{u}=\bigcup_{j=1}^{\infty} \partial B_{r_{j}}(0)$ the orientation such that $u^{i}=u^{+}=j+1$ and $u^{e}=u^{-}=j$ on $\partial B_{r_{j}}(0)$.

Let $\left(a_{j}\right) \subset \mathbb{R}$ be a bounded sequence, and let

$$
\boldsymbol{A}(x):=a(|x|) \frac{x}{|x|}, \quad \text { with } \quad a(\rho):=\sum_{j=1}^{\infty} a_{j} \chi_{\left[r_{j}, r_{j-1}\right)}(\rho), \rho \in(0,1) .
$$

We have that $\boldsymbol{A} \in L^{\infty}\left(\Omega, \mathbb{R}^{2}\right), \operatorname{Tr}^{i}\left(\boldsymbol{A}, J_{u}\right)=a_{j+1}, \operatorname{Tr}^{e}\left(\boldsymbol{A}, J_{u}\right)=a_{j}$ on $\partial B_{r_{j}}$, and

$$
\begin{gathered}
\operatorname{div} \boldsymbol{A}=\frac{a(|x|)}{|x|} \mathcal{L}^{2}+\sum_{j=1}^{\infty}\left(a_{j+1}-a_{j}\right) \mathcal{H}^{1}\left\llcorner\partial B_{r_{j}},\right. \\
|\operatorname{div} \boldsymbol{A}|(\Omega) \leq\|\boldsymbol{A}\|_{\infty} \int_{\Omega} \frac{1}{|x|} d x+2 \pi \sum_{j=1}^{\infty}\left|a_{j+1}-a_{j}\right| r_{j}<+\infty,
\end{gathered}
$$

so that $\boldsymbol{A} \in \mathcal{D} \mathcal{M}^{\infty}(\Omega)$. On the other hand, if we choose a sequence $\left(a_{j}\right)$ such that $\left|a_{j}\right| \geq c>0$ for every $j \in \mathbb{N}$, we have that

$$
\int_{J_{u}} u^{-}\left|\operatorname{Tr}^{i, e}\left(\boldsymbol{A}, J_{u}\right)\right| d \mathcal{H}^{1} \geq 2 \pi c \sum_{j=1}^{\infty} j r_{j}=+\infty .
$$

We conclude this example observing that, with the choice $a_{j}=(-1)^{j}$, we have also that

$$
\int_{J_{u}} u^{ \pm}\left|\operatorname{Tr}^{i}\left(\boldsymbol{A}, J_{u}\right)-\operatorname{Tr}^{e}\left(\boldsymbol{A}, J_{u}\right)\right| d \mathcal{H}^{1}=+\infty .
$$

We collect here the main features of the truncation operator that will be useful to generalize to $u \in B V(\Omega) \cap L^{1}(\Omega,|\operatorname{div} \boldsymbol{A}|)$ properties valid in $B V(\Omega) \cap L^{\infty}(\Omega)$.

Proposition 3.4 (Properties of the truncated functions). For every $k>0$, let

$$
T_{k}(s):=\max \{\min \{s, k\},-k\}, \quad s \in \mathbb{R} .
$$

Let $u \in B V(\Omega)$ and let $\lambda: \Omega \rightarrow[0,1]$ be a Borel function. Then the following hold.

(i) $T_{k}\left(u^{ \pm}\right)=\left[T_{k}(u)\right]^{ \pm} \rightarrow u^{ \pm},\left[T_{k}(u)\right]^{\lambda} \rightarrow u^{\lambda}, \mathcal{H}^{N-1}$-a.e. in $\Omega$;

(ii) $\left|D T_{k}(u)\right| \leq|D u|$ in the sense of measures, for every $k>0$; 
(iii) $\left|\left[T_{k}(u)\right]^{ \pm}\right| \leq\left|u^{ \pm}\right|$for every $k>0$, hence

$$
\left|T_{k}\left(u^{\lambda}\right)\right| \leq(1-\lambda)\left|u^{-}\right|+\lambda\left|u^{+}\right| \quad \forall k>0 ;
$$

(iv) if $u \in B V(\Omega) \cap L^{1}(\Omega,|\operatorname{div} \boldsymbol{A}|)$, then $T_{k}\left(u^{\lambda}\right) \rightarrow u^{\lambda}$ in $L^{1}(\Omega,|\operatorname{div} \boldsymbol{A}|)$.

Proof. The proof of (i) can be found in [3, Theorem 4.34(a)].

The inequality in (ii) is a consequence of the fact that $T_{k}$ is a 1-Lipschitz function (see the first part of the proof of Theorem 3.96 in [3]).

The inequalities in (iii) follow from $\left|T_{k}(s)\right| \leq|s|$ and the equalities in (i), whereas (iv) follows from (iii), Lemma 3.2 , and Lebesgue's Dominated Convergence Theorem.

\section{Definition AND BASIC PROPERTIES OF PAIRINGS}

Definition 4.1 (Generalized pairing). Given a vector field $\boldsymbol{A} \in \mathcal{D M}^{\infty}(\Omega)$ and a Borel function $\lambda: \Omega \rightarrow[0,1]$, for every $u \in B V(\Omega) \cap L^{1}(\Omega,|\operatorname{div} \boldsymbol{A}|)$ the $\lambda$-pairing between $\boldsymbol{A}$ and $D u$ is the distribution $(\boldsymbol{A}, D u)_{\lambda}: C_{c}^{\infty}(\Omega) \rightarrow \mathbb{R}$ acting as

$$
\left\langle(\boldsymbol{A}, D u)_{\lambda}, \varphi\right\rangle:=-\int_{\Omega} u^{\lambda} \varphi d(\operatorname{div} \boldsymbol{A})-\int_{\Omega} u \boldsymbol{A} \cdot \nabla \varphi d x, \quad \varphi \in C_{c}^{\infty}(\Omega) .
$$

Remark 4.2. The standard pairing

$$
\left\langle(\boldsymbol{A}, D u)_{*}, \varphi\right\rangle:=-\int_{\Omega} u^{*} \varphi d \operatorname{div} \boldsymbol{A}-\int_{\Omega} u \boldsymbol{A} \cdot \nabla \varphi d x
$$

introduced in [6], and deeply studied in recent years (see e.g. [10, [19] and the references therein), is the $\lambda$-pairing corresponding to the constant selection $\lambda(x)=\frac{1}{2}$ for every $x \in \Omega$.

Remark 4.3. The definition of generalized pairing and the properties proved in the rest of the paper can be extended straightforwardly to vector fields $\boldsymbol{A} \in \mathcal{D} \mathcal{M}_{\mathrm{loc}}^{\infty}(\Omega)$ and functions $u \in B V_{\text {loc }}(\Omega) \cap L_{\text {loc }}^{1}(\Omega,|\operatorname{div} \boldsymbol{A}|)$.

Clearly, the change of pointwise values of $u$ may just affect the behavior of the pairing on the jump set $J_{u}$ of $u$. More precisely, the following basic properties hold.

Proposition 4.4. Let $\boldsymbol{A} \in \mathcal{D} \mathcal{M}^{\infty}(\Omega), u \in B V(\Omega) \cap L^{1}(\Omega,|\operatorname{div} \boldsymbol{A}|)$, and $\lambda: \Omega \rightarrow[0,1]$ be a Borel function. Then $(\boldsymbol{A}, D u)_{\lambda}$ is a Radon measure in $\Omega$, and the equations

$$
\begin{gathered}
\operatorname{div}(u \boldsymbol{A})=u^{\lambda} \operatorname{div} \boldsymbol{A}+(\boldsymbol{A}, D u)_{\lambda}, \\
(\boldsymbol{A}, D u)_{\lambda}=(\boldsymbol{A}, D u)_{*}+\left(\frac{1}{2}-\lambda\right)\left(u^{+}-u^{-}\right) \operatorname{div} \boldsymbol{A}\left\llcorner J_{u},\right.
\end{gathered}
$$

hold in the sense of measures in $\Omega$. Moreover, $(\boldsymbol{A}, D u)_{\lambda}$ is absolutely continuous with respect to $|D u|$, and

$$
\left|(\boldsymbol{A}, D u)_{\lambda}\right| \leq\|\boldsymbol{A}\|_{\infty}|D u|
$$

In what follows we will write

$$
(\boldsymbol{A}, D u)_{\lambda}=\theta_{\lambda}(\boldsymbol{A}, D u, x)|D u|,
$$

where $\theta_{\lambda}(\boldsymbol{A}, D u, \cdot)$ denotes the Radon-Nikodým derivative of $(\boldsymbol{A}, D u)_{\lambda}$ with respect to $|D u|$. 
Proof. Assume, in addition, that $u \in B V(\Omega) \cap L^{\infty}(\Omega)$. In this case the fact that $(\boldsymbol{A}, D u)_{\lambda}$ is a Radon measure, and the validity of (19) are straightforward consequences of the fact that the distribution

$$
\langle\operatorname{div}(u \boldsymbol{A}), \varphi\rangle=\int_{\Omega} u \boldsymbol{A} \cdot \nabla \varphi d x, \quad \varphi \in C_{c}^{\infty}(\Omega)
$$

is a Radon measure in $\Omega$ (see [10]). Moreover, we have that

$$
\begin{aligned}
(\boldsymbol{A}, D u)_{\lambda} & =-u^{*} \operatorname{div} \boldsymbol{A}+\operatorname{div}(u \boldsymbol{A})+\left(\frac{1}{2}-\lambda\right)\left(u^{+}-u^{-}\right) \operatorname{div} \boldsymbol{A}\left\llcorner J_{u}\right. \\
& =(\boldsymbol{A}, D u)_{*}+\left(\frac{1}{2}-\lambda\right)\left(u^{+}-u^{-}\right) \operatorname{div} \boldsymbol{A}\left\llcorner J_{u} .\right.
\end{aligned}
$$

From [34, Proposition 3.5] (see in particular formula (3.9) there), we have that

$$
\left|(\boldsymbol{A}, D u)_{0}\right|,\left|(\boldsymbol{A}, D u)_{1}\right| \leq\|\boldsymbol{A}\|_{\infty}|D u| .
$$

Since $(\boldsymbol{A}, D u)_{\lambda}=(1-\lambda)(\boldsymbol{A}, D u)_{0}+\lambda(\boldsymbol{A}, D u)_{1}$, (21) follows.

Consider now the general case $u \in B V(\Omega) \cap L^{1}(\Omega,|\operatorname{div} \boldsymbol{A}|)$. Let $u_{k}:=T_{k}(u)$ be the sequence of truncated functions. By Proposition [3.4(i) and (iv), we have that $\left(u_{k}\right)^{\lambda} \rightarrow u^{\lambda}$ $\mathcal{H}^{N-1}$-a.e. in $\Omega$ and in $L^{1}(\Omega,|\operatorname{div} \boldsymbol{A}|)$. Hence, we can pass to the limit in

$$
\left\langle\left(\boldsymbol{A}, D u_{k}\right)_{\lambda}, \varphi\right\rangle=-\int_{\Omega}\left(u_{k}\right)^{\lambda} \varphi d(\operatorname{div} \boldsymbol{A})-\int_{\Omega} u_{k} \boldsymbol{A} \cdot \nabla \varphi d x
$$

and obtain that $(\boldsymbol{A}, D u)_{\lambda}$ is the weak* limit of $\left(\boldsymbol{A}, D u_{k}\right)_{\lambda}$ in the sense of measures, so that (19) follows. Since, by the estimate (21) and Proposition 3.4(ii), we have that

$$
\left|\left(\boldsymbol{A}, D u_{k}\right)_{\lambda}\right|(\Omega) \leq\|\boldsymbol{A}\|_{\infty}\left|D u_{k}\right|(\Omega) \leq\|\boldsymbol{A}\|_{\infty}|D u|(\Omega), \quad \forall k \in \mathbb{N},
$$

we conclude that (19), (20) and (21) hold in the sense of measures.

Remark 4.5. In the last part of the proof of Proposition 4.4 we have shown that, for every $\boldsymbol{A} \in \mathcal{D M}^{\infty}(\Omega)$ and every $u \in B V(\Omega) \cap L^{1}(\Omega,|\operatorname{div} \boldsymbol{A}|)$, the pairing $(\boldsymbol{A}, D u)_{\lambda}$ is the weak* limit, in the sense of measures, of the sequence $\left(\boldsymbol{A}, D T_{k}(u)\right)_{\lambda}$.

Remark 4.6. Since $(u+v)^{+} \leq u^{+}+v^{+}$and $(u+v)^{-} \geq u^{-}+v^{-}$, with possibly strict inequalities, the map $u \mapsto(\boldsymbol{A}, D u)_{\lambda}$ is not linear, in general. On the other hand, the map $u \mapsto u^{*}$ is linear, hence the standard pairing is linear with respect to $u$. More precisely, the $\lambda$-pairing is linear if and only if $(\boldsymbol{A}, D u)_{\lambda}=(\boldsymbol{A}, D u)_{*}$ for every $u \in B V\left(\mathbb{R}^{N}\right) \cap L^{\infty}\left(\mathbb{R}^{N}\right)$. Indeed, for every $u \in B V\left(\mathbb{R}^{N}\right) \cap L^{\infty}\left(\mathbb{R}^{N}\right)$ we have that

$$
\begin{aligned}
(\boldsymbol{A}, D u)_{\lambda}+(\boldsymbol{A}, D(-u))_{\lambda} & =\left(\frac{1}{2}-\lambda\right)\left[u^{+}-u^{-}+(-u)^{+}-(-u)^{-}\right] \operatorname{div} \boldsymbol{A}\left\llcorner J_{u}\right. \\
& =2\left(\frac{1}{2}-\lambda\right)\left(u^{+}-u^{-}\right) \operatorname{div} \boldsymbol{A}\left\llcorner J_{u} .\right.
\end{aligned}
$$

Hence, if there exists $u \in B V\left(\mathbb{R}^{N}\right) \cap L^{\infty}\left(\mathbb{R}^{N}\right)$ such that $(\boldsymbol{A}, D u)_{\lambda} \neq(\boldsymbol{A}, D u)_{*}$, then the claim follows from (20).

Using (20), and the results of Theorem 3.3 in [19], we are able to compute explicitly the diffuse part $(\boldsymbol{A}, D u)_{\lambda}^{d}$, the absolutely continuous part $(\boldsymbol{A}, D u)_{\lambda}^{a}$, and the jump part $(\boldsymbol{A}, D u)_{\lambda}^{j}$ of the generalized pairing. 
Proposition 4.7. Let $\boldsymbol{A} \in \mathcal{D M}^{\infty}(\Omega)$ and $u \in B V(\Omega) \cap L^{1}(\Omega,|\operatorname{div} \boldsymbol{A}|)$. Then the diffuse, the absolutely continuous and the jump part of the measure $(\boldsymbol{A}, D u)_{\lambda}$ are respectively

$$
\begin{gathered}
(\boldsymbol{A}, D u)_{\lambda}^{d}=(\boldsymbol{A}, D u)_{*}^{d}, \quad(\boldsymbol{A}, D u)_{\lambda}^{a}=\boldsymbol{A} \cdot \nabla u \mathcal{L}^{N}, \\
(\boldsymbol{A}, D u)_{\lambda}^{j}=\left[(1-\lambda) \operatorname{Tr}^{i}\left(\boldsymbol{A}, J_{u}\right)+\lambda \operatorname{Tr}^{e}\left(\boldsymbol{A}, J_{u}\right)\right]\left(u^{+}-u^{-}\right) \mathcal{H}^{N-1}\left\llcorner J_{u},\right.
\end{gathered}
$$

where $\operatorname{Tr}^{i}\left(\boldsymbol{A}, J_{u}\right)$ and $\operatorname{Tr}^{e}\left(\boldsymbol{A}, J_{u}\right)$ are the normal traces corresponding to the orientation of $J_{u}$ such that $u^{+}=u^{i}$.

Proof. By (20),$(\boldsymbol{A}, D u)_{\lambda}$ and $(\boldsymbol{A}, D u)_{*}^{d}$ may differ only on $J_{u}$, hence $(\boldsymbol{A}, D u)_{\lambda}^{d}=(\boldsymbol{A}, D u)_{*}^{d}$. Moreover, by Theorem 3.2 in [10], $(\boldsymbol{A}, D u)_{\lambda}^{a}=(\boldsymbol{A}, D u)_{*}^{a}=\boldsymbol{A} \cdot \nabla u \mathcal{L}^{N}$.

Concerning the jump part $(\boldsymbol{A}, D u)_{\lambda}^{j}$, by (21), we already know that it is concentrated on $J_{u}$. Denoting by $\alpha^{i}:=\operatorname{Tr}^{i}\left(\boldsymbol{A}, J_{u}\right)$ and $\alpha^{e}:=\operatorname{Tr}^{e}\left(\boldsymbol{A}, J_{u}\right)$, by Theorem 3.3 in [19] we already know that

$$
(\boldsymbol{A}, D u)_{*}^{j}=\frac{\alpha^{i}+\alpha^{e}}{2}\left(u^{+}-u^{-}\right) \mathcal{H}^{N-1}\left\llcorner J_{u} .\right.
$$

Finally, by (20) and (14), we conclude that

$$
\begin{aligned}
(\boldsymbol{A}, D u)_{\lambda}^{j} & =(\boldsymbol{A}, D u)_{*}^{j}+\left(\frac{1}{2}-\lambda\right)\left(u^{+}-u^{-}\right) \operatorname{div} \boldsymbol{A}\left\llcorner J_{u}\right. \\
& =\frac{\alpha^{i}+\alpha^{e}}{2}\left(u^{+}-u^{-}\right) \mathcal{H}^{N-1}\left\llcorner J_{u}+\left(\frac{1}{2}-\lambda\right)\left(u^{+}-u^{-}\right)\left(\alpha^{i}-\alpha^{e}\right) \mathcal{H}^{N-1}\left\llcorner J_{u}\right.\right. \\
& =\left[(1-\lambda) \alpha^{i}+\lambda \alpha^{e}\right]\left(u^{+}-u^{-}\right) \mathcal{H}^{N-1}\left\llcorner J_{u} .\right.
\end{aligned}
$$

Remark 4.8 (The pairing trivializes on $W^{1,1}$ ). From Proposition 4.7, we have that

$$
(\boldsymbol{A}, D u)_{\lambda}=(\boldsymbol{A}, D u)_{*}=\boldsymbol{A} \cdot \nabla u \mathcal{L}^{N}, \quad \forall u \in W^{1,1}(\Omega) \cap L^{\infty}(\Omega) .
$$

Remark 4.9 ( $B V$ vector fields). If $\boldsymbol{A} \in B V\left(\Omega, \mathbb{R}^{N}\right) \cap L^{\infty}\left(\Omega, \mathbb{R}^{N}\right)$, then clearly $\boldsymbol{A} \in$ $\mathcal{D} \mathcal{M}^{\infty}(\Omega)$ and

$$
\operatorname{Tr}^{i, e}\left(\boldsymbol{A}, J_{u}\right)=\boldsymbol{A}_{J_{u}}^{i, e} \cdot \nu_{u}, \quad \mathcal{H}^{N-1} \text {-a.e. in } J_{u},
$$

where $\boldsymbol{A}_{J_{u}}^{i, e}$ are the traces of $\boldsymbol{A}$ on $J_{u}$ in the sense of $B V$ (see [3, Theorem 3.77]). Hence, the jump part of $(\boldsymbol{A}, D u)_{\lambda}$ can be written as

$$
(\boldsymbol{A}, D u)_{\lambda}^{j}=\left[(1-\lambda) \boldsymbol{A}_{J_{u}}^{i}+\lambda \boldsymbol{A}_{J_{u}}^{e}\right] \cdot D^{j} u .
$$

Remark 4.10 (The pairing trivializes for continuous vector fields). If $\boldsymbol{A} \in C\left(\Omega, \mathbb{R}^{N}\right)$, then by [19, Theorem 3.3] and [16, Theorem 3.7] it holds

$$
(\boldsymbol{A}, D u)_{\lambda}=\boldsymbol{A} \cdot D u, \quad \forall u \in B V(\Omega) \cap L^{1}(\Omega,|\operatorname{div} \boldsymbol{A}|) .
$$

The following result is an improvement of Proposition 4.15 in [19, Theorem 1.2 in [10] and Lemma 2.2 in 6 .

Proposition 4.11 (Approximation by $C^{\infty}$ fields). Let $\boldsymbol{A} \in \mathcal{D} \mathcal{M}^{\infty}(\Omega)$. Then there exists a sequence $\left(\boldsymbol{A}_{k}\right)_{k}$ in $C^{\infty}\left(\Omega, \mathbb{R}^{N}\right) \cap L^{\infty}\left(\Omega, \mathbb{R}^{N}\right)$ satisfying the following properties.

(i) $\boldsymbol{A}_{k}-\boldsymbol{A} \rightarrow 0$ in $L^{1}\left(\Omega, \mathbb{R}^{N}\right), \int_{\Omega}\left|\operatorname{div} \boldsymbol{A}_{k}\right| d x \rightarrow|\operatorname{div} \boldsymbol{A}|(\Omega)$, and $\left(\boldsymbol{A}_{k}\right)_{k}$ is uniformly bounded.

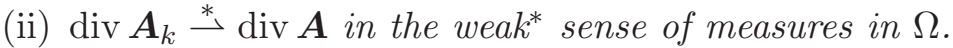


(iii) For every oriented countably $\mathcal{H}^{N-1}$-rectifiable set $\Sigma \subset \Omega$ it holds

$$
\lim _{k \rightarrow+\infty}\left\langle\operatorname{Tr}^{i, e}\left(\boldsymbol{A}_{k}, \Sigma\right), \varphi\right\rangle=\left\langle\operatorname{Tr}^{*}(\boldsymbol{A}, \Sigma), \varphi\right\rangle \quad \forall \varphi \in C_{c}(\Omega),
$$

where $\operatorname{Tr}^{*}(\boldsymbol{A}, \Sigma):=\left[\operatorname{Tr}^{i}(\boldsymbol{A}, \Sigma)+\operatorname{Tr}^{e}(\boldsymbol{A}, \Sigma)\right] / 2$.

Moreover, for every $u \in B V(\Omega) \cap L^{\infty}(\Omega)$, it holds

(iv) $\left(\boldsymbol{A}_{k}, D u\right)_{*} \stackrel{*}{\rightarrow}(\boldsymbol{A}, D u)_{*}$ locally in the weak $k^{*}$ sense of measures in $\Omega$;

(v) the sequence $\theta\left(\boldsymbol{A}_{k}, D u ; \cdot\right)$ weakly* converges in $L^{\infty}(\Omega,|D u|)$ to $\theta(\boldsymbol{A}, D u ; \cdot)$, where $\theta(\boldsymbol{A}, D u ; \cdot)$ is the Radon-Nikodým derivative of $(\boldsymbol{A}, D u)_{*}$ with respect to $|D u|$.

Remark 4.12. It is not difficult to show that a similar approximation result holds also for $\boldsymbol{A} \in \mathcal{D} \mathcal{M}_{\mathrm{loc}}^{\infty}(\Omega)$ with a sequence $\left(\boldsymbol{A}_{k}\right)$ in $C^{\infty}\left(\Omega, \mathbb{R}^{N}\right)$.

Proof. (i) This part is proved in [10, Theorem 1.2]. We just recall, for later use, that for every $k$ the vector field $\boldsymbol{A}_{k}$ is of the form

$$
\boldsymbol{A}_{k}=\sum_{i=1}^{\infty} \rho_{\varepsilon_{i}} *\left(\boldsymbol{A} \varphi_{i}\right)
$$

where $\left(\varphi_{i}\right)$ is a partition of unity subordinate to a locally finite covering of $\Omega$ depending on $k$ and, for every $i, \varepsilon_{i} \in(0,1 / k)$ is chosen in such a way that

$$
\int_{\Omega}\left|\rho_{\varepsilon_{i}} *\left(\boldsymbol{A} \cdot \nabla \varphi_{i}\right)-\boldsymbol{A} \cdot \nabla \varphi_{i}\right| d x \leq \frac{1}{k 2^{i}}
$$

(see [10], formula (1.8)).

(ii) From (i) we have that

$$
\lim _{k \rightarrow+\infty} \int_{\Omega} \boldsymbol{A}_{k} \cdot \nabla \varphi d x=\int_{\Omega} \boldsymbol{A} \cdot \nabla \varphi d x \quad \forall \varphi \in C_{c}^{1}(\Omega)
$$

hence (ii) follows from the density of $C_{c}^{1}(\Omega)$ in $C_{0}(\Omega)$ in the norm of $L^{\infty}(\Omega)$ and the bound $\sup _{k} \int_{\Omega}\left|\operatorname{div} \boldsymbol{A}_{k}\right| d x<+\infty$.

(iii) As a first step we prove that, for every $u \in B V(\Omega) \cap L^{\infty}(\Omega)$,

$$
\lim _{k \rightarrow+\infty} \int_{\Omega} u \varphi \operatorname{div} \boldsymbol{A}_{k} d x=\int_{\Omega} u^{*} \varphi d \operatorname{div} \boldsymbol{A}, \quad \forall \varphi \in C_{c}(\Omega) .
$$

Specifically, from the definition (23) of $\boldsymbol{A}_{k}$ and the identity $\sum_{i} \nabla \varphi_{i}=0$ we have that

$$
\operatorname{div} \boldsymbol{A}_{k}=\sum_{i} \rho_{\varepsilon_{i}} *\left(\varphi_{i} \operatorname{div} \boldsymbol{A}\right)+\sum_{i}\left[\rho_{\varepsilon_{i}} *\left(\boldsymbol{A} \cdot \nabla \varphi_{i}\right)-\boldsymbol{A} \cdot \nabla \varphi_{i}\right] .
$$

From the estimate (24) we have that

$$
\left|\sum_{i} \int_{\Omega} u \varphi\left[\rho_{\varepsilon_{i}} *\left(\boldsymbol{A} \cdot \nabla \varphi_{i}\right)-\boldsymbol{A} \cdot \nabla \varphi_{i}\right] d x\right|<\frac{1}{k}\|\varphi\|_{\infty}\|u\|_{\infty},
$$

and hence, to prove (25), it is enough to show that

$$
\lim _{k \rightarrow+\infty} \sum_{i} \int_{\Omega} u \varphi \rho_{\varepsilon_{i}} *\left(\varphi_{i} \operatorname{div} \boldsymbol{A}\right)=\int_{\Omega} u^{*} \varphi d \operatorname{div} \boldsymbol{A} .
$$

On the other hand,

$$
\sum_{i} \int_{\Omega} u \varphi \rho_{\varepsilon_{i}} *\left(\varphi_{i} \operatorname{div} \boldsymbol{A}\right)=\sum_{i} \int_{\Omega} \rho_{\varepsilon_{i}} *(u \varphi) \varphi_{i} d \operatorname{div} \boldsymbol{A}
$$


hence (26) follows by observing that the functions $\rho_{\varepsilon_{i}} *(u \varphi)$ converge pointwise $\mathcal{H}^{N-1}$-a.e. in $\Omega$ to $u^{*} \varphi$, so that

$$
u^{*} \varphi-\sum_{i} \varphi_{i} \rho_{\varepsilon_{i}} *(u \varphi)=\sum_{i} \varphi_{i}\left[u^{*} \varphi-\rho_{\varepsilon_{i}} *(u \varphi)\right] \rightarrow 0, \quad|\operatorname{div} \boldsymbol{A}| \text {-a.e. in } \Omega .
$$

We remark that, as a consequence of (25), if $E \Subset \Omega$ is a set of finite perimeter, then

$$
\lim _{k \rightarrow+\infty} \int_{\Omega} \chi_{E} \varphi \operatorname{div} \boldsymbol{A}_{k} d x=\int_{\Omega} \chi_{E}^{*} \varphi d \operatorname{div} \boldsymbol{A}, \quad \forall \varphi \in C_{c}(\Omega) .
$$

Let us now prove (iii). Let $\omega \Subset \Omega$ be a set of class $C^{1}$. By the definition (12) of normal traces, by (i), (ii) and (27), for every $\varphi \in C_{c}^{\infty}(\Omega)$ we have that

$$
\begin{aligned}
\left\langle\operatorname{Tr}\left(\boldsymbol{A}_{k}, \partial \omega\right), \varphi\right\rangle & =\int_{\omega} \boldsymbol{A}_{k} \cdot \nabla \varphi d x+\int_{\omega} \varphi \operatorname{div} \boldsymbol{A}_{k} d x \\
& =\int_{\Omega} \chi_{\omega} \boldsymbol{A}_{k} \cdot \nabla \varphi d x+\int_{\Omega} \chi_{\omega} \varphi \operatorname{div} \boldsymbol{A}_{k} d x,
\end{aligned}
$$

so that

$$
\begin{aligned}
\lim _{k \rightarrow+\infty}\left\langle\operatorname{Tr}\left(\boldsymbol{A}_{k}, \partial \omega\right), \varphi\right\rangle & =\int_{\Omega} \chi_{\omega} \boldsymbol{A} \cdot \nabla \varphi d x+\int_{\Omega} \chi_{\omega}^{*} \varphi d \operatorname{div} \boldsymbol{A} \\
& =\int_{\omega} \boldsymbol{A} \cdot \nabla \varphi d x+\int_{\omega} \varphi d \operatorname{div} \boldsymbol{A}+\frac{1}{2} \int_{\partial \omega} \varphi d \operatorname{div} \boldsymbol{A} \\
& =\langle\operatorname{Tr}(\boldsymbol{A}, \partial \omega), \varphi\rangle+\frac{1}{2}\langle\operatorname{div} \boldsymbol{A}\llcorner\partial \omega, \varphi\rangle .
\end{aligned}
$$

Hence, by (14), we have proved that

$$
\lim _{k \rightarrow+\infty} \operatorname{Tr}^{e}\left(\boldsymbol{A}_{k}, \partial \omega\right)=\operatorname{Tr}^{e}(\boldsymbol{A}, \partial \omega)+\frac{1}{2}\left[\operatorname{Tr}^{i}(\boldsymbol{A}, \partial \omega)-\operatorname{Tr}^{e}(\boldsymbol{A}, \partial \omega)\right]=\operatorname{Tr}^{*}(\boldsymbol{A}, \partial \omega),
$$

in the sense of distributions. Using the arguments of Section 2.4, this relation can be extended to the countably $\mathcal{H}^{N-1}$-rectifiable set $\Sigma$. By a density argument as in (ii), this relation hold for every $\varphi \in C_{c}(\Omega)$, hence (iii) holds true for $\operatorname{Tr}^{e}\left(\boldsymbol{A}_{k}, \Sigma\right)$. Finally, a similar computation holds for $\operatorname{Tr}^{i}\left(\boldsymbol{A}_{k}, \Sigma\right)$.

(iv) Using the passage to the limit in (25) we obtain straightforwardly

$$
\begin{aligned}
\lim _{k \rightarrow+\infty}\left\langle\left(\boldsymbol{A}_{k}, D u\right)_{*}, \varphi\right\rangle & =\lim _{k \rightarrow+\infty}\left[-\int_{\Omega} u^{*} \varphi \operatorname{div} \boldsymbol{A}_{k} d x-\int_{\Omega} u \boldsymbol{A}_{k} \cdot \nabla \varphi d x\right] \\
& =-\int_{\Omega} u^{*} \varphi d \operatorname{div} \boldsymbol{A}-\int_{\Omega} u \boldsymbol{A} \cdot \nabla \varphi d x \\
& =\left\langle(\boldsymbol{A}, D u)_{*}, \varphi\right\rangle
\end{aligned}
$$

for every $\varphi \in C_{c}^{1}(\Omega)$. The validity of this relation for $\varphi \in C_{c}(\Omega)$ follows from (21) and the fact that the sequence $\left(\boldsymbol{A}_{k}\right)$ is bounded in $L^{\infty}\left(\Omega, \mathbb{R}^{N}\right)$.

(v) Using the definition (22) of the density $\theta$, we have that, for every $\varphi \in C_{c}(\Omega)$,

$$
\begin{aligned}
\lim _{k \rightarrow+\infty} \int_{\Omega} \theta\left(\boldsymbol{A}_{k}, D u, x\right) \varphi(x) d|D u| & =\lim _{k \rightarrow+\infty}\left\langle\left(\boldsymbol{A}_{k}, D u\right)_{*}, \varphi\right\rangle \\
& =\left\langle(\boldsymbol{A}, D u)_{*}, \varphi\right\rangle=\int_{\Omega} \theta(\boldsymbol{A}, D u, x) \varphi(x) d|D u| .
\end{aligned}
$$

Since, by (21) and (22), the sequence $\left(\theta\left(\boldsymbol{A}_{k}, D u, \cdot\right)\right)$ is bounded in $L^{\infty}(\Omega,|D u|)$, then (v) follows. 


\section{COAREA FORMUla FOR GENERALIZED PAIRINGS}

This section is devoted to the proof of the coarea formula for the $\lambda$-pairing, and a related slicing result for its density $\theta_{\lambda}$.

Theorem 5.1 (Coarea formula). Let $\boldsymbol{A} \in \mathcal{D} \mathcal{M}^{\infty}(\Omega)$ and let $u \in B V(\Omega) \cap L^{1}(\Omega,|\operatorname{div} \boldsymbol{A}|)$. Then $\chi_{\{u>t\}} \in B V(\Omega)$ for $\mathcal{L}^{1}$-a.e. $t \in \mathbb{R}$, and

$$
\left\langle(\boldsymbol{A}, D u)_{\lambda}, \varphi\right\rangle=\int_{\mathbb{R}}\left\langle\left(\boldsymbol{A}, D \chi_{\{u>t\}}\right)_{\lambda}, \varphi\right\rangle d t, \quad \forall \varphi \in C_{0}(\Omega) .
$$

Proof. Since $(\boldsymbol{A}, D u)_{\lambda}$ and $\left(\boldsymbol{A}, D \chi_{\{u>t\}}\right)_{\lambda}$ are measures in $\Omega$ for $\mathcal{L}^{1}$-a.e. $t \in \mathbb{R}$, it is enough to prove (28) for $\varphi \in C_{c}^{\infty}(\Omega)$.

Let us first consider the case $u \in L^{\infty}(\Omega)$. By possibly replacing $u$ with $u+\|u\|_{\infty}$, it is not restrictive to assume that $u \geq 0$. Given a test function $\varphi \in C_{c}^{\infty}(\Omega)$, we have that

$$
\begin{aligned}
\int_{\mathbb{R}} & \left\langle\left(\boldsymbol{A}, D \chi_{\{u>t\}}\right)_{\lambda}, \varphi\right\rangle d t \\
& =-\int_{0}^{+\infty}\left(\int_{\Omega} \chi_{\{u>t\}}^{\lambda} \varphi d \operatorname{div} \boldsymbol{A}\right) d t-\int_{0}^{+\infty}\left(\int_{\Omega} \chi_{\{u>t\}} \boldsymbol{A} \cdot \nabla \varphi d x\right) d t \\
& =-\int_{0}^{+\infty}\left(\int_{\Omega} \chi_{\{u>t\}}^{\lambda} \varphi d \operatorname{div} \boldsymbol{A}\right) d t-\int_{\Omega} u \boldsymbol{A} \cdot \nabla \varphi d x .
\end{aligned}
$$

Moreover, by [20, Lemma 2.2], we have that, for $\mathcal{L}^{1}$-a.e. $t \in \mathbb{R}$, there exists a Borel set $N_{t} \subset \Omega$, with $\mathcal{H}^{N-1}\left(N_{t}\right)=0$, such that

$$
\forall x \in \Omega \backslash N_{t}: \quad \chi_{\{u>t\}}^{-}(x)=\chi_{\left\{u^{-}>t\right\}}(x), \quad \chi_{\{u>t\}}^{+}(x)=\chi_{\left\{u^{+}>t\right\}}(x),
$$

so that, since $|\operatorname{div} \boldsymbol{A}| \ll \mathcal{H}^{N-1}$, we obtain that

$$
\chi_{\{u>t\}}^{\lambda}(x)=(1-\lambda(x)) \chi_{\left\{u^{-}>t\right\}}(x)+\lambda(x) \chi_{\left\{u^{+}>t\right\}}(x), \quad \text { for }|\operatorname{div} \boldsymbol{A}| \text {-a.e. } x \in \Omega .
$$

Hence, we get

$$
\begin{aligned}
& \int_{0}^{+\infty}\left(\int_{\Omega} \chi_{\{u>t\}}^{\lambda} \varphi d \operatorname{div} \boldsymbol{A}\right) d t \\
& =\int_{0}^{+\infty}\left(\int_{\Omega}\left[(1-\lambda) \chi_{\left\{u^{-}>t\right\}}+\lambda \chi_{\left\{u^{+}>t\right\}}\right] \varphi d \operatorname{div} \boldsymbol{A}\right) d t \\
& =\int_{\Omega}(1-\lambda) \varphi\left(\int_{0}^{+\infty} \chi_{\left\{u^{-}>t\right\}} d t\right) d \operatorname{div} \boldsymbol{A}+\int_{\Omega} \lambda \varphi\left(\int_{0}^{+\infty} \chi_{\left\{u^{+}>t\right\}} d t\right) d \operatorname{div} \boldsymbol{A} \\
& =\int_{\Omega} u^{\lambda} \varphi d \operatorname{div} \boldsymbol{A} .
\end{aligned}
$$

As a consequence, from (29), (30) and the definition (18) of $(\boldsymbol{A}, D u)_{\lambda}$, we conclude that (28) holds for every test function $\varphi \in C_{c}^{\infty}(\Omega)$ and for every $u \in B V\left(\mathbb{R}^{N}\right) \cap L^{\infty}\left(\mathbb{R}^{N}\right)$.

Finally, the general case $u \in B V(\Omega) \cap L^{1}(\Omega,|\operatorname{div} \boldsymbol{A}|)$ follows applying the previous step to the truncated functions $u_{k}:=T_{k}(u)$. Specifically, (28) gives, for every $k>0$,

$$
\left\langle\left(\boldsymbol{A}, D u_{k}\right)_{\lambda}, \varphi\right\rangle=\int_{\mathbb{R}}\left\langle\left(\boldsymbol{A}, D \chi_{\left\{u_{k}>t\right\}}\right)_{\lambda}, \varphi\right\rangle d t, \quad \forall \varphi \in C_{c}^{1}(\Omega) .
$$


By Remark 4.5, the left-hand side of (31) converges to $\left\langle(\boldsymbol{A}, D u)_{\lambda}, \varphi\right\rangle$. On the other hand, since

$$
\begin{gathered}
\{u>t\}=\left\{u_{k}>t\right\}, \quad D \chi_{\{u>t\}}=D \chi_{\left\{u_{k}>t\right\}}, \quad \forall t \in[-k, k), \\
D \chi_{\left\{u_{k}>t\right\}}=0, \quad \forall t \in \mathbb{R} \backslash[-k, k),
\end{gathered}
$$

the right-hand side in (31) is equal to

$$
\int_{-k}^{k}\left\langle\left(A, D \chi_{\{u>t\}}\right)_{\lambda}, \varphi\right\rangle d t
$$

By the estimate (21) we have that

$$
\left|\left\langle\left(\boldsymbol{A}, D \chi_{\{u>t\}}\right)_{\lambda}, \varphi\right\rangle\right| \leq 2\|\varphi\|_{\infty}\|\boldsymbol{A}\|_{\infty}\left|D \chi_{\{u>t\}}\right|(\Omega),
$$

and hence, by the coarea formula in $B V$ and the Lebesgue Dominated Convergence Theorem, the integral in (32) converges to the right-hand side of (28) as $k \rightarrow+\infty$.

Proposition 5.2. Let $\boldsymbol{A} \in \mathcal{D M}^{\infty}(\Omega)$ and $u \in B V(\Omega) \cap L^{\infty}(\Omega)$. Then

(33) for $\mathcal{L}^{1}$-a.e. $t \in \mathbb{R}: \quad \theta_{\lambda}(\boldsymbol{A}, D u, x)=\theta_{\lambda}\left(\boldsymbol{A}, D \chi_{\{u>t\}}, x\right) \quad$ for $\left|D \chi_{\{u>t\}}\right|$-a.e. $x \in \Omega$.

Proof. Thanks to Proposition 4.11 (iv), the proof can be done following the lines of 6 , Proposition 2.7(iii)]. For the reader's convenience, we recall here the main points.

Given two real numbers $a<b$, the function $v:=\max \{\min \{u, b\}, a\}$ satisfies

$$
\begin{gathered}
\{u>t\}=\{v>t\}, \quad D \chi_{\{u>t\}}=D \chi_{\{v>t\}}, \quad \forall t \in[a, b), \\
D \chi_{\{v>t\}}=0, \quad \forall t<a, t \geq b .
\end{gathered}
$$

Since

$$
\frac{d D u}{d|D u|}=\frac{d D \chi_{\{u>t\}}}{d\left|D \chi_{\{u>t\}}\right|}, \quad\left|D \chi_{\{u>t\}}\right| \text {-a.e. in } \Omega
$$

(see [25, §4.1.4, Theorem 2(i)]), we deduce that

$$
\frac{d D u}{d|D u|}=\frac{d D v}{d|D v|} \quad|D v| \text {-a.e. in } \Omega \text {. }
$$

Let $\left(\boldsymbol{A}_{k}\right) \subset C^{\infty}\left(\Omega, \mathbb{R}^{N}\right) \cap L^{\infty}\left(\Omega, \mathbb{R}^{N}\right)$ be the sequence of smooth vector fields approximating $\boldsymbol{A}$ as in Proposition [4.11. Since, by [6, Proposition 2.3], we have

$$
\theta\left(\boldsymbol{A}_{k}, D u, x\right)=\boldsymbol{A}_{k}(x) \cdot \frac{d D u}{d|D u|}(x)=\boldsymbol{A}_{k}(x) \cdot \frac{d D v}{d|D v|}(x)=\theta\left(\boldsymbol{A}_{k}, D v, x\right) \quad|D v| \text {-a.e. in } \Omega,
$$

then, from Proposition 4.11 (v) and by the uniqueness of the limit in the $L^{\infty}(\Omega,|D v|)$ weak* topology, we obtain that

$$
\theta(\boldsymbol{A}, D u, x)=\theta(\boldsymbol{A}, D v, x) \quad|D v| \text {-a.e. in } \Omega .
$$

Recalling the definition (22) of $\theta_{\lambda}$ and the relation (20), we conclude that

$$
\theta_{\lambda}(\boldsymbol{A}, D u, x)=\theta_{\lambda}(\boldsymbol{A}, D v, x) \quad|D v| \text {-a.e. in } \Omega \text {. }
$$

Specifically, $\theta_{\lambda}(\boldsymbol{A}, D u, x)=\theta(\boldsymbol{A}, D u, x)=\theta(\boldsymbol{A}, D v, x)=\theta_{\lambda}(\boldsymbol{A}, D v, x)$ for $\left|D^{d} v\right|$-a.e. $x \in$ $\Omega$, whereas, by Proposition 4.7 (and using the notations therein) and the inclusion $J_{v} \subset J_{u}$, $\theta_{\lambda}(\boldsymbol{A}, D u, x)=(1-\lambda) \operatorname{Tr}^{i}\left(\boldsymbol{A}, J_{u}\right)+\lambda \operatorname{Tr}^{e}\left(\boldsymbol{A}, J_{u}\right)=\theta_{\lambda}(\boldsymbol{A}, D v, x)$ for $\left|D^{j} v\right|$-a.e. $x \in \Omega$. 
Given $\varphi \in C_{c}^{\infty}(\Omega)$, let us compute $\left\langle(\boldsymbol{A}, D v)_{\lambda}, \varphi\right\rangle$. By the definition of $\theta_{\lambda}(\boldsymbol{A}, D v, x)$, equality (35), the coarea formula in BV (see [3, Theorem 3.40]) and (34) it holds

$$
\begin{aligned}
\left\langle(\boldsymbol{A}, D v)_{\lambda}, \varphi\right\rangle & =\int_{\Omega} \theta_{\lambda}(\boldsymbol{A}, D v, x) \varphi(x) d|D v| \\
& =\int_{\Omega} \theta_{\lambda}(\boldsymbol{A}, D u, x) \varphi(x) d|D v| \\
& =\int_{a}^{b} d t \int_{\Omega} \theta_{\lambda}(\boldsymbol{A}, D u, x) \varphi(x) d\left|D \chi_{\{u>t\}}\right| .
\end{aligned}
$$

On the other hand, by the coarea formula (28) and (34), it holds

$$
\begin{aligned}
\left\langle(\boldsymbol{A}, D v)_{\lambda}, \varphi\right\rangle & =\int_{\mathbb{R}}\left\langle\left(\boldsymbol{A}, D \chi_{\{v>t\}}\right)_{\lambda}, \varphi\right\rangle d t \\
& =\int_{a}^{b}\left\langle\left(\boldsymbol{A}, D \chi_{\{u>t\}}\right)_{\lambda}, \varphi\right\rangle d t \\
& =\int_{a}^{b} d t \int_{\Omega} \theta_{\lambda}\left(\boldsymbol{A}, D \chi_{\{u>t\}}, x\right) \varphi(x) d\left|D \chi_{\{u>t\}}\right| .
\end{aligned}
$$

Comparing (36) with (37), we finally conclude that, for every $a<b$,

$$
\int_{a}^{b} d t \int_{\Omega} \theta_{\lambda}(\boldsymbol{A}, D u, x) \varphi(x) d\left|D \chi_{\{u>t\}}\right|=\int_{a}^{b} d t \int_{\Omega} \theta_{\lambda}\left(\boldsymbol{A}, D \chi_{\{u>t\}}, x\right) \varphi(x) d\left|D \chi_{\{u>t\}}\right|,
$$

so that (33) follows.

\section{Chain Rule, Leibniz and Gauss-Green formulas for generalized Pairings}

In this section we show that some relevant formulas, proved in [19] for the standard pairing, remain valid for general $\lambda$-pairings.

Proposition 6.1 (Chain Rule). Let $\boldsymbol{A} \in \mathcal{D M}^{\infty}(\Omega)$ and let $u \in B V(\Omega) \cap L^{\infty}(\Omega)$. Let $h: \mathbb{R} \rightarrow \mathbb{R}$ be a Lipschitz function. Then it holds:

(i) $(\boldsymbol{A}, D[h(u)])_{\lambda}^{d}=(\boldsymbol{A}, D[h(u)])_{*}^{d}$, and $(\boldsymbol{A}, D[h(u)])_{\lambda}^{a}=h^{\prime}(\widetilde{u}) \boldsymbol{A} \cdot \nabla u \mathcal{L}^{N}$.

Moreover, if $h$ is non-decreasing, then

(ii) $(\boldsymbol{A}, D[h(u)])_{\lambda}^{j}=\frac{h\left(u^{+}\right)-h\left(u^{-}\right)}{u^{+}-u^{-}}(\boldsymbol{A}, D u)_{\lambda}^{j}$;

(iii) $\theta_{\lambda}(\boldsymbol{A}, D[h(u)], x)=\theta_{\lambda}(\boldsymbol{A}, D u, x)$, for $|D[h(u)]|$-a.e. $x \in \Omega$.

The same characterization holds if $u \in B V_{\mathrm{loc}}(\Omega) \cap L_{\mathrm{loc}}^{\infty}(\Omega)$ and $h: I \rightarrow \mathbb{R}$ is a locally Lipschitz function such that $u(\Omega) \Subset I$.

Proof. Although the proof is essentially the same of [19, Proposition 4.5], for the sake of completeness we prefer to illustrate it in some detail.

One of the main ingredients is the Chain Rule Formula for $B V$ functions (see 3, Theorem 3.99]):

$D^{d}[h(u)]=h^{\prime}(\widetilde{u}) D^{d} u, \quad D^{a}[h(u)]=h^{\prime}(u) \nabla u \mathcal{L}^{N}, \quad D^{j}[h(u)]=\left[h\left(u^{i}\right)-h\left(u^{e}\right)\right] \nu_{u} \mathcal{H}^{N-1}\left\llcorner J_{u}\right.$.

Statement (i) easily follows from the first two relations above and Proposition 4.7.

Concerning (ii), we have that $[h(u)]^{i, e}=h\left(u^{i, e}\right)$ (see [3. Proposition 3.69(c)]). Moreover, since $h$ is non-decreasing, also the relations $[h(u)]^{ \pm}=h\left(u^{ \pm}\right)$hold true, and hence (ii) follows again from Proposition 4.7. 
Let us prove (iii). If $h$ is strictly increasing, we can follow the proof of [6. Proposition 2.8]. Specifically, $\{u>t\}=\{h(u)>h(t)\}$ for every $t \in \mathbb{R}$, hence

$$
D \chi_{\{u>t\}}=D \chi_{\{h(u)>h(t)\}}, \quad \forall t \in \mathbb{R} .
$$

From Proposition 5.2, for $\mathcal{L}^{1}$-a.e. $t \in \mathbb{R}$ it holds

$$
\theta_{\lambda}(\boldsymbol{A}, D u, x)=\theta_{\lambda}\left(\boldsymbol{A}, D \chi_{\{u>t\}}, x\right)=\theta_{\lambda}\left(\boldsymbol{A}, D \chi_{\{h(u)>h(t)\}}, x\right)=\theta_{\lambda}(\boldsymbol{A}, D[h(u)], x)
$$

for $\left|D \chi_{\{u>t\}}\right|$-a.e. $x \in \Omega$, and (iii) follows.

If $h$ is non-decreasing, we can adapt the proof of [29, Proposition 2.7]. Specifically, let $h_{\varepsilon}(t):=h(t)+\varepsilon t$, so that $h_{\varepsilon}$ is strictly increasing for every $\varepsilon>0$. Since

$$
\left[h_{\varepsilon}(u)\right]^{\lambda}=(1-\lambda) h_{\varepsilon}\left(u^{-}\right)+\lambda h_{\varepsilon}\left(u^{+}\right)=[h(u)]^{\lambda}+\varepsilon u^{\lambda},
$$

by the previous step we deduce that

$$
(\boldsymbol{A}, D[h(u)])_{\lambda}+\varepsilon(\boldsymbol{A}, D u)_{\lambda}=\left(\boldsymbol{A}, D\left[h_{\varepsilon}(u)\right]\right)_{\lambda}=\theta_{\lambda}(\boldsymbol{A}, D u, x)\left|D\left[h_{\varepsilon}(u)\right]\right| .
$$

On the other hand,

$$
D\left[h_{\varepsilon}(u)\right]=\left[h^{\prime}(\widetilde{u})+\varepsilon\right] D^{d} u+\left[h\left(u^{i}\right)-h\left(u^{e}\right)+\varepsilon\left(u^{i}-u^{e}\right)\right] D^{j} u=D[h(u)]+\varepsilon D u,
$$

hence, passing to the limit in (38) as $\varepsilon \rightarrow 0$, we deduce that

$$
(\boldsymbol{A}, D[h(u)])_{\lambda}=\theta_{\lambda}(\boldsymbol{A}, D u, x)|D[h(u)]| \quad \text { as measures in } \Omega,
$$

and (iii) follows.

Proposition 6.2 (Leibniz formula). Let $\boldsymbol{A} \in \mathcal{D M}^{\infty}(\Omega)$ and $u, v \in B V(\Omega) \cap L^{\infty}(\Omega)$. Then, choosing on $J_{u}$ the orientation such that $u^{+}=u^{i}$, it holds

$$
\begin{gathered}
(v \boldsymbol{A}, D u)_{\lambda}^{d}=v^{\lambda}(\boldsymbol{A}, D u)_{\lambda}^{d}=v^{*}(\boldsymbol{A}, D u)_{*}^{d}, \\
(v \boldsymbol{A}, D u)_{\lambda}^{j}=\left[(1-\lambda) \operatorname{Tr}^{i}\left(\boldsymbol{A}, J_{u}\right) v^{i}+\lambda \operatorname{Tr}^{e}\left(\boldsymbol{A}, J_{u}\right) v^{e}\right]\left(u^{+}-u^{-}\right) \mathcal{H}^{N-1}\left\llcorner J_{u} .\right.
\end{gathered}
$$

Proof. By [19, Proposition 4.9], denoting $\alpha^{i}:=\operatorname{Tr}^{i}\left(\boldsymbol{A}, J_{u}\right)$ and $\alpha^{e}:=\operatorname{Tr}^{e}\left(\boldsymbol{A}, J_{u}\right)$ we have that

$$
\begin{gathered}
(v \boldsymbol{A}, D u)_{*}^{d}=v^{*}(\boldsymbol{A}, D u)_{*}^{d}, \\
(v \boldsymbol{A}, D u)_{*}^{j}=\frac{\alpha^{i} v^{i}+\alpha^{e} v^{e}}{2}\left(u^{+}-u^{-}\right) \mathcal{H}^{N-1}\left\llcorner J_{u},\right.
\end{gathered}
$$

hence (39) follows from (41) and Proposition 4.7.

From the representation formulas (14) and Proposition 2.6, we get

$$
\operatorname{div}(v \boldsymbol{A})\left\llcorner J_{u}=\left[\operatorname{Tr}^{i}\left(v \boldsymbol{A}, J_{u}\right)-\operatorname{Tr}^{e}\left(v \boldsymbol{A}, J_{u}\right)\right] \mathcal{H}^{N-1}\left\llcorner J_{u}=\left(v^{i} \alpha^{i}-v^{e} \alpha^{e}\right) \mathcal{H}^{N-1}\left\llcorner J_{u},\right.\right.\right.
$$

hence, from (42), we obtain

$$
(v \boldsymbol{A}, D u)_{\lambda}^{j}=\left[\frac{\alpha^{i} v^{i}+\alpha^{e} v^{e}}{2}+\left(\frac{1}{2}-\lambda\right)\left(v^{i} \alpha^{i}-v^{e} \alpha^{e}\right)\right]\left(u^{+}-u^{-}\right) \mathcal{H}^{N-1}\left\llcorner J_{u},\right.
$$

that is (40) holds.

In the last part of this section we will prove a generalized Gauss-Green formula for vector fields $\boldsymbol{A} \in \mathcal{D} \mathcal{M}^{\infty}\left(\mathbb{R}^{N}\right)$ on a set $E \subset \mathbb{R}^{N}$ of finite perimeter, generalizing the analogous result for the standard pairing proved in [19, Theorem 5.1].

Using the conventions of Section 2.4, we will assume that the generalized normal vector on $\partial^{*} E$ coincides $\mathcal{H}^{N-1}$-a.e. on $\partial^{*} E$ with the measure-theoretic interior unit normal vector to $E$. 
Theorem 6.3 (Gauss-Green). Let $\boldsymbol{A} \in \mathcal{D} \mathcal{M}^{\infty}\left(\mathbb{R}^{N}\right)$ and $u \in B V\left(\mathbb{R}^{N}\right) \cap L^{1}\left(\mathbb{R}^{N},|\operatorname{div} \boldsymbol{A}|\right)$. Let $E \subset \mathbb{R}^{N}$ be a bounded set with finite perimeter, and assume that the traces $u^{e}, u^{i}$ of $u$ on $\partial^{*} E$ belong to $L^{1}\left(\partial^{*} E, \mathcal{H}^{N-1}\left\llcorner\partial^{*} E\right)\right.$. Then the following Gauss-Green formulas hold:

$$
\begin{gathered}
\int_{E^{1}} u^{\lambda} d \operatorname{div} \boldsymbol{A}+\int_{E^{1}}(\boldsymbol{A}, D u)_{\lambda}=-\int_{\partial^{*} E} \operatorname{Tr}^{i}\left(\boldsymbol{A}, \partial^{*} E\right) u^{i} d \mathcal{H}^{N-1}, \\
\int_{E^{1} \cup \partial^{*} E} u^{\lambda} d \operatorname{div} \boldsymbol{A}+\int_{E^{1} \cup \partial^{*} E}(\boldsymbol{A}, D u)_{\lambda}=-\int_{\partial^{*} E} \operatorname{Tr}^{e}\left(\boldsymbol{A}, \partial^{*} E\right) u^{e} d \mathcal{H}^{N-1},
\end{gathered}
$$

where $E^{1}$ is the measure theoretic interior of $E$ and $\partial^{*} E$ is oriented with respect to the interior unit normal vector.

Proof. We recall that, by Lemma 3.2, $u^{\lambda} \in L_{\text {loc }}^{1}\left(\mathbb{R}^{N},|\operatorname{div} \boldsymbol{A}|\right)$. Recalling (20), we have that

$$
\int_{E^{1}}(\boldsymbol{A}, D u)_{\lambda}=\int_{E^{1}}(\boldsymbol{A}, D u)_{*}+\int_{E^{1}}\left(\frac{1}{2}-\lambda\right)\left(u^{+}-u^{-}\right) d \operatorname{div} \boldsymbol{A} .
$$

On the other hand, by the definition (11) of $u^{\lambda}$, it holds

$$
\int_{E^{1}} u^{\lambda} d \operatorname{div} \boldsymbol{A}=\int_{E^{1}} u^{*} d \operatorname{div} \boldsymbol{A}-\int_{E^{1}}\left(\frac{1}{2}-\lambda\right)\left(u^{+}-u^{-}\right) d \operatorname{div} \boldsymbol{A}
$$

so that (43) follows from the Gauss-Green formula for the standard pairing proved in [19, Theorem 5.1]. The validity of (44) can be checked in a very similar way.

\section{Semicontinuity Results}

In this section we consider the pairing as a function in $B V$

$$
B V(\Omega) \cap L^{1}(\Omega,|\operatorname{div} \boldsymbol{A}|) \ni u \mapsto(\boldsymbol{A}, D u)_{\lambda} \in \mathcal{M}_{b}(\Omega),
$$

where $\mathcal{M}_{b}(\Omega)$ denotes the space of finite Borel measures on $\Omega$ (see (21)).

Our aim is to characterize the selections $\lambda: \Omega \rightarrow[0,1]$ such that the above map is lower (resp. upper) semicontinuous, meaning that, if $\left(u_{n}\right) \subset B V(\Omega) \cap L^{1}(\Omega,|\operatorname{div} \boldsymbol{A}|)$ is a sequence converging to a function $u \in B V(\Omega) \cap L^{1}(\Omega,|\operatorname{div} \boldsymbol{A}|$ ) (in a suitable way), then

$$
\begin{gathered}
\left\langle(\boldsymbol{A}, D u)_{\lambda}, \varphi\right\rangle \leq \liminf _{n}\left\langle\left(\boldsymbol{A}, D u_{n}\right)_{\lambda}, \varphi\right\rangle \quad \forall \varphi \in C_{c}^{\infty}(\Omega), \varphi \geq 0 \\
\left(\operatorname{resp} . \quad\left\langle(\boldsymbol{A}, D u)_{\lambda}, \varphi\right\rangle \geq \underset{n}{\limsup }\left\langle\left(\boldsymbol{A}, D u_{n}\right)_{\lambda}, \varphi\right\rangle \quad \forall \varphi \in C_{c}^{\infty}(\Omega), \varphi \geq 0\right) .
\end{gathered}
$$

Since $(\boldsymbol{A}, D u)_{\lambda}$ is affected by the pointwise value of $u$, the correct notion of convergence in $B V$ seems to be the strict one (see e.g. [3, Definition 3.14]).

Definition 7.1. The sequence $\left(u_{n}\right) \subset B V(\Omega)$ strictly converges to $u \in B V(\Omega)$ if $\left(u_{n}\right)$ converges to $u$ in $L^{1}(\Omega)$ and the total variations $\left|D u_{n}\right|(\Omega)$ converge to $|D u|(\Omega)$.

We recall a recent result concerning the pointwise behavior of strictly converging sequences.

Proposition 7.2. Every sequence $\left(u_{n}\right)$ strictly convergent in $B V(\Omega)$ to u admits a subsequence $\left(u_{n_{k}}\right)$ such that for $\mathcal{H}^{N-1}$-a.e. $x \in \Omega$

$$
u^{-}(x) \leq \liminf _{k} u_{n_{k}}^{-}(x) \leq \underset{k}{\limsup } u_{n_{k}}^{+}(x) \leq u^{+}(x) .
$$

In particular, $\lim _{k} \widetilde{u}_{n_{k}}(x)=\widetilde{u}(x)$ for $\mathcal{H}^{N-1}$-a.e. $x \in \Omega \backslash J_{u}$.

Proof. See [28], Theorem 3.2, and Corollary 3.3. 
Combining Proposition 7.2 with Theorem 3.3 in [7, we obtain the following approximation result.

Proposition 7.3. Let $\Omega \subset \mathbb{R}^{N}$ be a bounded open set, and let $u \in B V(\Omega)$. Then there exist two sequences $\left(u_{n}\right),\left(v_{n}\right) \subset W^{1,1}(\Omega)$ such that:

(a) for every $n \in \mathbb{N}, \widetilde{v}_{n} \leq u^{-}$and $u^{+} \leq \widetilde{u}_{n} \mathcal{H}^{N-1}$-a.e. in $\Omega$;

(b) $u_{n} \rightarrow u, v_{n} \rightarrow u$ strictly in $B V$;

(c) $\widetilde{u}_{n}(x) \rightarrow u^{+}(x)$ and $\widetilde{v}_{n}(x) \rightarrow u^{-}(x)$ for $\mathcal{H}^{N-1}$-a.e. $x \in \Omega$.

If, in addition, $u \in L^{\infty}(\Omega)$, then the above sequences are bounded in $L^{\infty}(\Omega)$.

Proof. From Theorem 3.3 in [7], there exists a sequence $\left(u_{n}\right) \subset W^{1,1}(\Omega)$, strictly convergent to $u$, and such that $\widetilde{u}_{n} \geq u^{+} \mathcal{H}^{N-1}$-a.e. in $\Omega$, for every $n \in \mathbb{N}$. Moreover, if $u$ is bounded, then this sequence is bounded in $L^{\infty}(\Omega)$. By Proposition 7.2 , we can extract a subsequence (not relabeled) such that

$$
\underset{n}{\limsup } u_{n}^{+}(x) \leq u^{+}(x), \quad \text { for } \mathcal{H}^{N-1} \text {-a.e. } x \in \Omega .
$$

On the other hand, the inequality $\widetilde{u}_{n} \geq u^{+}$gives

$$
\liminf _{n} u_{n}^{+}(x) \geq u^{+}(x), \quad \text { for } \mathcal{H}^{N-1} \text {-a.e. } x \in \Omega,
$$

hence the assertion for $\left(u_{n}\right)$ follows. The construction of $\left(v_{n}\right)$ can be done in a similar way.

In order to state the semicontinuity results, a more piece of notation is needed. Given a vector field $\boldsymbol{A} \in \mathcal{D} \mathcal{M}^{\infty}(\Omega)$, let us denote by $\Omega_{\boldsymbol{A}}$ the set of points $x \in \Omega$ such that $x$ belongs to the support of $\operatorname{div} \boldsymbol{A}$ (i.e. $|\operatorname{div} \boldsymbol{A}|\left(B_{r}(x) \cap \Omega\right)>0$ for every $r>0$ ), and the limit

$$
\psi_{\boldsymbol{A}}(x):=\lim _{r \rightarrow 0} \frac{\operatorname{div} \boldsymbol{A}\left(B_{r}(x)\right)}{|\operatorname{div} \boldsymbol{A}|\left(B_{r}(x)\right)}
$$

exists in $\mathbb{R}$, with $\left|\psi_{\boldsymbol{A}}(x)\right|=1$. If we extend $\psi_{\boldsymbol{A}}=0$ in $\Omega \backslash \Omega_{\boldsymbol{A}}$, we have that $\psi_{\boldsymbol{A}} \in$ $L^{1}(\Omega,|\operatorname{div} \boldsymbol{A}|)$ and the polar decomposition $\operatorname{div} \boldsymbol{A}=\psi_{\boldsymbol{A}}|\operatorname{div} \boldsymbol{A}|$ holds. Moreover, if we define the sets

$$
\Omega_{\boldsymbol{A}}^{+}:=\left\{x \in \Omega_{\boldsymbol{A}}: \psi_{\boldsymbol{A}}(x)=1\right\}, \quad \Omega_{\boldsymbol{A}}^{-}:=\left\{x \in \Omega_{\boldsymbol{A}}: \psi_{\boldsymbol{A}}(x)=-1\right\},
$$

then $(\operatorname{div} \boldsymbol{A})^{+}=\operatorname{div} \boldsymbol{A}\left\llcorner\Omega_{\boldsymbol{A}}^{+}\right.$and $(\operatorname{div} \boldsymbol{A})^{-}=-\operatorname{div} \boldsymbol{A}\left\llcorner\Omega_{\boldsymbol{A}}^{-}\right.$.

Let $\Theta_{\boldsymbol{A}}$ be the jump set of the measure $|\operatorname{div} \boldsymbol{A}|$ (see Proposition 2.3). Since $\Theta_{\boldsymbol{A}}$ is $\sigma$-finite with respect to $\mathcal{H}^{N-1}$, then there exists a countably $\mathcal{H}^{N-1}$-rectifiable Borel set $\Theta_{\boldsymbol{A}}^{r} \subseteq \Theta_{\boldsymbol{A}}$ such that $\Theta_{\boldsymbol{A}}^{u}:=\Theta_{\boldsymbol{A}} \backslash \Theta_{\boldsymbol{A}}^{r}$ is purely $\mathcal{H}^{N-1}$-unrectifiable (i.e. $\mathcal{H}^{N-1}\left(\Theta_{\boldsymbol{A}}^{u} \cap \Sigma\right)=0$ for every countably $\mathcal{H}^{N-1}$-rectifiable set $\Sigma$, see [3, Definition 2.64 and Proposition 2.76]).

Let us define the families of selections

$\Lambda_{\mathrm{lsc}}:=\left\{\lambda: \Omega \rightarrow[0,1]\right.$ Borel: $\lambda=0 \mathcal{H}^{N-1}$-a.e. in $\Theta_{\boldsymbol{A}}^{r} \cap \Omega_{\boldsymbol{A}}^{-}, \lambda=1 \mathcal{H}^{N-1}$-a.e. in $\left.\Theta_{\boldsymbol{A}}^{r} \cap \Omega_{\boldsymbol{A}}^{+}\right\}$ $\Lambda_{\text {usc }}:=\left\{\lambda: \Omega \rightarrow[0,1]\right.$ Borel: $\lambda=1 \mathcal{H}^{N-1}$-a.e. in $\Theta_{\boldsymbol{A}}^{r} \cap \Omega_{\boldsymbol{A}}^{-}, \lambda=0 \mathcal{H}^{N-1}$-a.e. in $\left.\Theta_{\boldsymbol{A}}^{r} \cap \Omega_{\boldsymbol{A}}^{+}\right\}$.

These families satisfy the following extremality properties.

Lemma 7.4. Given $\boldsymbol{A} \in \mathcal{D} \mathcal{M}^{\infty}(\Omega), u \in B V(\Omega) \cap L^{1}(\Omega,|\operatorname{div} \boldsymbol{A}|), \varphi \in C_{0}(\Omega), \varphi \geq 0$, then for every Borel function $\lambda: \Omega \rightarrow[0,1]$ it holds

$$
\int_{\Omega_{\boldsymbol{A}}^{+}} u^{\lambda} \varphi d \operatorname{div} \boldsymbol{A} \leq \int_{\Omega_{\boldsymbol{A}}^{+}} u^{+} \varphi d \operatorname{div} \boldsymbol{A}, \quad \int_{\Omega_{\boldsymbol{A}}^{-}} u^{\lambda} \varphi d \operatorname{div} \boldsymbol{A} \leq \int_{\Omega_{\boldsymbol{A}}^{-}} u^{-} \varphi d \operatorname{div} \boldsymbol{A},
$$

with equality if $\lambda \in \Lambda_{\mathrm{lsc}}$. 
Similarly,

$$
\int_{\Omega_{\boldsymbol{A}}^{+}} u^{\lambda} \varphi d \operatorname{div} \boldsymbol{A} \geq \int_{\Omega_{\boldsymbol{A}}^{+}} u^{-} \varphi d \operatorname{div} \boldsymbol{A}, \quad \int_{\Omega_{\boldsymbol{A}}^{-}} u^{\lambda} \varphi d \operatorname{div} \boldsymbol{A} \geq \int_{\Omega_{\boldsymbol{A}}^{-}} u^{+} \varphi d \operatorname{div} \boldsymbol{A},
$$

with equality if $\lambda \in \Lambda_{\text {usc }}$.

Proof. Let us prove the claim only for the first inequality in (47), the other being similar.

Since, by the very definition of $\Omega_{\boldsymbol{A}}^{+}$,

$$
\int_{\Omega_{\boldsymbol{A}}^{+}} u^{\lambda} \varphi d \operatorname{div} \boldsymbol{A}=\int_{\Omega_{\boldsymbol{A}}^{+}} u^{\lambda} \varphi d|\operatorname{div} \boldsymbol{A}|
$$

and $u^{\lambda} \leq u^{+} \mathcal{H}^{N-1}$-a.e. in $\Omega$, the first inequality in (47) follows.

Let $\lambda \in \Lambda_{\mathrm{lsc}}$ and let us prove that equality holds in the first inequality in (47). Let us decompose the set $\Omega_{\boldsymbol{A}}^{+}$, defined in (46), as the union of the disjoint sets

$$
\Omega_{\boldsymbol{A}}^{+} \backslash J_{u}, \quad \Omega_{\boldsymbol{A}}^{+} \cap\left(J_{u} \cap \Theta_{\boldsymbol{A}}\right), \quad \Omega_{\boldsymbol{A}}^{+} \cap\left(J_{u} \backslash \Theta_{\boldsymbol{A}}\right),
$$

that, in turn, coincide up to sets of $\mathcal{H}^{N-1}$-measure zero respectively with

$$
\Omega_{\boldsymbol{A}}^{+} \backslash S_{u}, \quad \Omega_{\boldsymbol{A}}^{+} \cap \Theta_{\boldsymbol{A}}^{r} \cap J_{u}, \quad\left(\Omega_{\boldsymbol{A}}^{+} \backslash \Theta_{\boldsymbol{A}}\right) \cap J_{u} .
$$

Observe that $u^{\lambda}=\widetilde{u} \mathcal{H}^{N-1}$-a.e. (hence $|\operatorname{div} \boldsymbol{A}|$-a.e.) in $\Omega_{\boldsymbol{A}}^{+} \backslash S_{u}, u^{\lambda}=u^{+} \mathcal{H}^{N-1}$-a.e. in $\Omega_{\boldsymbol{A}}^{+} \cap \Theta_{\boldsymbol{A}}^{r}$, and, by Proposition 2.3, $|\operatorname{div} \boldsymbol{A}|\left(\left(\Omega_{\boldsymbol{A}}^{+} \backslash \Theta_{\boldsymbol{A}}\right) \cap J_{u}\right)=0$ Hence,

$$
\begin{aligned}
\int_{\Omega_{\boldsymbol{A}}^{+}} u^{\lambda} \varphi d \operatorname{div} \boldsymbol{A} & =\int_{\Omega_{\boldsymbol{A}}^{+}} u^{\lambda} \varphi d|\operatorname{div} \boldsymbol{A}| \\
& =\int_{\Omega_{\boldsymbol{A}}^{+} \backslash S_{u}} \widetilde{u} \varphi d|\operatorname{div} \boldsymbol{A}|+\int_{\Omega_{\boldsymbol{A}}^{+} \cap \Theta_{\boldsymbol{A}}^{r} \cap J_{u}} u^{+} \varphi d|\operatorname{div} \boldsymbol{A}| \\
& =\int_{\Omega_{\boldsymbol{A}}^{+}} u^{+} \varphi d \operatorname{div} \boldsymbol{A} .
\end{aligned}
$$

Corollary 7.5. Given $\boldsymbol{A} \in \mathcal{D}^{\infty}(\Omega)$ and $u \in B V(\Omega) \cap L^{1}(\Omega,|\operatorname{div} \boldsymbol{A}|)$, it holds:

$$
\begin{array}{ll}
(\boldsymbol{A}, D u)_{\lambda}=-u^{+}(\operatorname{div} \boldsymbol{A})^{+}+u^{-}(\operatorname{div} \boldsymbol{A})^{-}+\operatorname{div}(u \boldsymbol{A}), & \forall \lambda \in \Lambda_{\mathrm{lsc}}, \\
(\boldsymbol{A}, D u)_{\lambda}=-u^{-}(\operatorname{div} \boldsymbol{A})^{+}+u^{+}(\operatorname{div} \boldsymbol{A})^{-}+\operatorname{div}(u \boldsymbol{A}), & \forall \lambda \in \Lambda_{\mathrm{usc}} .
\end{array}
$$

In particular,

$$
\begin{array}{ll}
(\boldsymbol{A}, D u)_{\lambda}=\min \left\{(\boldsymbol{A}, D u)_{0},(\boldsymbol{A}, D u)_{1}\right\}, & \forall \lambda \in \Lambda_{\mathrm{lsc}}, \\
(\boldsymbol{A}, D u)_{\lambda}=\max \left\{(\boldsymbol{A}, D u)_{0},(\boldsymbol{A}, D u)_{1}\right\}, & \forall \lambda \in \Lambda_{\mathrm{usc}} .
\end{array}
$$

Moreover, if the orientation of $J_{u}$ is chosen in such a way that $u^{+}=u^{i}$, then,

$$
\begin{array}{ll}
(\boldsymbol{A}, D u)_{\lambda}^{j}=\min \left\{\operatorname{Tr}^{i}\left(\boldsymbol{A}, J_{u}\right), \operatorname{Tr}^{e}\left(\boldsymbol{A}, J_{u}\right)\right\}\left(u^{+}-u^{-}\right) \mathcal{H}^{N-1}\left\llcorner J_{u},\right. & \forall \lambda \in \Lambda_{\mathrm{lsc}}, \\
(\boldsymbol{A}, D u)_{\lambda}^{j}=\max \left\{\operatorname{Tr}^{i}\left(\boldsymbol{A}, J_{u}\right), \operatorname{Tr}^{e}\left(\boldsymbol{A}, J_{u}\right)\right\}\left(u^{+}-u^{-}\right) \mathcal{H}^{N-1}\left\llcorner J_{u},\right. & \forall \lambda \in \Lambda_{\mathrm{usc}} .
\end{array}
$$

Proof. The first part is a direct consequence of the equality case in Lemma 7.4.

Let us prove (51). To simplify the notation, let

$$
\mu:=\operatorname{div} \boldsymbol{A}, \quad \nu:=\min \left\{(\boldsymbol{A}, D u)_{0},(\boldsymbol{A}, D u)_{1}\right\} .
$$

Since $(\boldsymbol{A}, D u)_{0}=-u^{-} \mu+\operatorname{div}(u \boldsymbol{A})$ and $(\boldsymbol{A}, D u)_{1}=-u^{+} \mu+\operatorname{div}(u \boldsymbol{A})$, by definition of minimum of two measures, for every Borel set $E \subset \Omega$ one has

$$
\nu(E)=\operatorname{div}(u \boldsymbol{A})(E)+\inf \left\{-u^{-} \mu^{+}\left(E_{0}\right)-u^{+} \mu^{+}\left(E_{1}\right)+u^{-} \mu^{-}\left(E_{0}\right)+u^{+} \mu^{-}\left(E_{1}\right)\right\},
$$


where the infimum is taken over the pairs $E_{0}, E_{1}$ of disjoint Borel sets such that $E=$ $E_{0} \cup E_{1}$. Setting $E^{-}:=E \cap \Omega_{\boldsymbol{A}}^{-}$and $E^{+}:=E \backslash E^{-}$, then $E \cap \Omega_{\boldsymbol{A}}^{+} \subset E^{+}$and

$$
\begin{gathered}
-u^{-} \mu^{+}\left(E_{0}\right)-u^{+} \mu^{+}\left(E_{1}\right) \geq-u^{+} \mu^{+}\left(E^{+}\right)=-u^{+} \mu^{+}(E), \\
u^{-} \mu^{-}\left(E_{0}\right)+u^{+} \mu^{-}\left(E_{1}\right) \geq u^{-} \mu^{-}\left(E^{-}\right)=u^{-} \mu^{-}(E),
\end{gathered}
$$

for every partition $\left\{E_{0}, E_{1}\right\}$ of $E$. Hence,

$$
\nu(E)=\operatorname{div}(u \boldsymbol{A})(E)-u^{+} \mu^{+}(E)+u^{-} \mu^{-}(E)=(\boldsymbol{A}, D u)_{\lambda}(E), \quad \forall \lambda \in \Lambda_{\mathrm{lsc}} .
$$

The proof of (52) is similar. Finally, (53) and (54) are consequences of (51) and (52), respectively, and Proposition 4.7.

Theorem 7.6. Let $\boldsymbol{A} \in \mathcal{D} \mathcal{M}^{\infty}(\Omega)$, and let $\lambda: \Omega \rightarrow[0,1]$ be a Borel function.

Then $\lambda \in \Lambda_{\mathrm{lsc}}$ if and only if, for every $u_{n}, u \in B V(\Omega)$ satisfying

(a) $u_{n} \rightarrow u$ strictly in $B V$,

(b) there exists $g \in L^{1}(\Omega,|\operatorname{div} \boldsymbol{A}|)$ such that, for every $n \in \mathbb{N},\left|u_{n}^{ \pm}\right| \leq g|\operatorname{div} \boldsymbol{A}|$-a.e. in $\Omega$,

it holds

$$
\left\langle(\boldsymbol{A}, D u)_{\lambda}, \varphi\right\rangle \leq \liminf _{n}\left\langle\left(\boldsymbol{A}, D u_{n}\right)_{\lambda}, \varphi\right\rangle \quad \forall \varphi \in C_{c}^{\infty}(\Omega), \varphi \geq 0 .
$$

Analogously, $\lambda \in \Lambda_{\mathrm{usc}}$ if and only if, for every $u_{n}, u \in B V(\Omega)$ satisfying (a), (b) it holds

$$
\left\langle(\boldsymbol{A}, D u)_{\lambda}, \varphi\right\rangle \geq \underset{n}{\limsup }\left\langle\left(\boldsymbol{A}, D u_{n}\right)_{\lambda}, \varphi\right\rangle \quad \forall \varphi \in C_{c}^{\infty}(\Omega), \varphi \geq 0 .
$$

Proof. Let us prove only the statement concerning the lower semicontinuity, the other being similar.

Let $\lambda \in \Lambda_{\text {lsc }}$, let $u_{n}, u \in B V(\Omega)$ satisfy (a), (b), and let us prove that the semicontinuity property in (55) holds. Let $\varphi \in C_{c}^{\infty}(\Omega), \varphi \geq 0$, and let $\left(u_{n_{k}}\right)$ be a subsequence such that

$$
\liminf _{n}\left\langle\left(\boldsymbol{A}, D u_{n}\right)_{\lambda}, \varphi\right\rangle=\lim _{k}\left\langle\left(\boldsymbol{A}, D u_{n_{k}}\right)_{\lambda}, \varphi\right\rangle,
$$

and (45) holds true (here we use (a) and Proposition 7.2).

From Lemma 7.4, assumption (b), Fatou's Lemma and the pointwise estimates (45) we have that

$$
\underset{k}{\limsup } \int_{\Omega_{\boldsymbol{A}}^{+}} u_{n_{k}}^{\lambda} \varphi d \operatorname{div} \boldsymbol{A} \leq \underset{k}{\limsup } \int_{\Omega_{\boldsymbol{A}}^{+}} u_{n_{k}}^{+} \varphi d \operatorname{div} \boldsymbol{A} \leq \int_{\Omega_{\boldsymbol{A}}^{+}} u^{+} \varphi d \operatorname{div} \boldsymbol{A} .
$$

Recalling that

$$
\int_{\Omega_{\boldsymbol{A}}^{-}} u_{n_{k}}^{\lambda} \varphi d \operatorname{div} \boldsymbol{A}=-\int_{\Omega_{\boldsymbol{A}}^{-}} u_{n_{k}}^{\lambda} \varphi d|\operatorname{div} \boldsymbol{A}|,
$$

the same argument gives

$$
\limsup _{k} \int_{\Omega_{\boldsymbol{A}}^{-}} u_{n_{k}}^{\lambda} \varphi d \operatorname{div} \boldsymbol{A} \leq \int_{\Omega_{\boldsymbol{A}}^{-}} u^{-} \varphi d \operatorname{div} \boldsymbol{A} .
$$

Since $|\operatorname{div} \boldsymbol{A}|\left(\Omega \backslash\left(\Omega_{\boldsymbol{A}}^{-} \cup \Omega_{\boldsymbol{A}}^{+}\right)\right)=0$, from (57), (58) and the equality case in (47) we get

(59) $\quad \limsup \int_{\Omega} u_{n_{k}}^{\lambda} \varphi d \operatorname{div} \boldsymbol{A} \leq \int_{\Omega_{\boldsymbol{A}}^{+}} u^{+} \varphi d \operatorname{div} \boldsymbol{A}+\int_{\Omega_{\boldsymbol{A}}^{-}} u^{-} \varphi d \operatorname{div} \boldsymbol{A}=\int_{\Omega} u^{\lambda} \varphi d \operatorname{div} \boldsymbol{A}$. 
Finally, from (59) and (a) we conclude that

$$
\begin{aligned}
& \liminf _{n}\left\langle\left(\boldsymbol{A}, D u_{n}\right)_{\lambda}, \varphi\right\rangle=-\underset{k}{\limsup }\left(\int_{\Omega} u_{n_{k}}^{\lambda} \varphi d \operatorname{div} A+\int_{\Omega} u_{n_{k}} \boldsymbol{A} \cdot \nabla \varphi d x\right) \\
& \geq-\int_{\Omega} u^{\lambda} \varphi d \operatorname{div} A-\int_{\Omega} u \boldsymbol{A} \cdot \nabla \varphi d x \\
& =\left\langle(\boldsymbol{A}, D u)_{\lambda}, \varphi\right\rangle,
\end{aligned}
$$

i.e., (55) holds true.

Assume now that (55) holds true for every $u_{n}, u \in B V(\Omega)$ satisfying (a), (b), and let us prove that $\lambda \in \Lambda_{\mathrm{lsc}}$. We claim that, under these assumptions,

$$
(\boldsymbol{A}, D u)_{\lambda} \leq(\boldsymbol{A}, D u)_{0}, \quad(\boldsymbol{A}, D u)_{\lambda} \leq(\boldsymbol{A}, D u)_{1}, \quad \forall u \in B V(\Omega) \cap L^{1}(\Omega,|\operatorname{div} \boldsymbol{A}|),
$$

in the sense of measures. By a truncation argument and Remark 4.5, it is enough to show that the above inequality holds for every $u \in B V(\Omega) \cap L^{\infty}(\Omega)$. Let $u \in B V(\Omega) \cap L^{\infty}(\Omega)$ and let $\left(u_{n}\right),\left(v_{n}\right) \subset W^{1,1}(\Omega) \cap L^{\infty}(\Omega)$ be the approximating sequences given by Proposition 7.3 . Observe that these sequences are bounded in $L^{\infty}(\Omega)$, so that they satisfy assumption (b). Since $\left(\widetilde{u}_{n}\right)$ converges to $u^{+}|\operatorname{div} \boldsymbol{A}|$-a.e. in $\Omega$ and, by (b), also in $L^{1}(\Omega,|\operatorname{div} \boldsymbol{A}|)$, for every test function $\varphi \in C_{c}^{\infty}(\Omega)$ we have that

$$
\begin{aligned}
\lim _{n}\left\langle\left(\boldsymbol{A}, D u_{n}\right)_{\lambda}, \varphi\right\rangle & =\lim _{n}\left(-\int_{\Omega} \widetilde{u}_{n} \varphi d \operatorname{div} \boldsymbol{A}-\int_{\Omega} u_{n} \boldsymbol{A} \cdot \nabla \varphi d x\right) \\
& =-\int_{\Omega} u^{+} \varphi d \operatorname{div} \boldsymbol{A}-\int_{\Omega} u \boldsymbol{A} \cdot \nabla \varphi d x=\left\langle(\boldsymbol{A}, D u)_{1}, \varphi\right\rangle,
\end{aligned}
$$

hence, by the semicontinuity assumption, if $\varphi \geq 0$,

$$
\left\langle(\boldsymbol{A}, D u)_{\lambda}, \varphi\right\rangle \leq \liminf _{n}\left\langle\left(\boldsymbol{A}, D u_{n}\right)_{\lambda}, \varphi\right\rangle=\left\langle(\boldsymbol{A}, D u)_{1}, \varphi\right\rangle .
$$

The same argument, using the sequence $\left(v_{n}\right)$, shows that

$$
\left\langle(\boldsymbol{A}, D u)_{\lambda}, \varphi\right\rangle \leq \liminf _{n}\left\langle\left(\boldsymbol{A}, D v_{n}\right)_{\lambda}, \varphi\right\rangle=\left\langle(\boldsymbol{A}, D u)_{0}, \varphi\right\rangle,
$$

so that (60) follows.

Let $\Omega^{\prime} \Subset \Omega$ be an open domain with $C^{1}$ boundary. From Proposition 4.7 we have that

$$
\left(\boldsymbol{A}, D \chi_{\Omega^{\prime}}\right)_{\lambda}=\left[(1-\lambda) \operatorname{Tr}^{i}\left(\boldsymbol{A}, \partial \Omega^{\prime}\right)+\lambda \operatorname{Tr}^{e}\left(\boldsymbol{A}, \partial \Omega^{\prime}\right)\right] \mathcal{H}^{N-1} \mathbf{L} \partial \Omega^{\prime},
$$

hence, the inequalities (60) give

$$
\left\{\begin{array}{l}
(1-\lambda)\left[\operatorname{Tr}^{i}\left(\boldsymbol{A}, \partial \Omega^{\prime}\right)-\operatorname{Tr}^{e}\left(\boldsymbol{A}, \partial \Omega^{\prime}\right)\right] \leq 0, \\
-\lambda\left[\operatorname{Tr}^{i}\left(\boldsymbol{A}, \partial \Omega^{\prime}\right)-\operatorname{Tr}^{e}\left(\boldsymbol{A}, \partial \Omega^{\prime}\right)\right] \leq 0,
\end{array} \quad \mathcal{H}^{N-1} \text {-a.e. on } \partial \Omega^{\prime} .\right.
$$

Let $\Sigma \subset \Omega$ be an oriented countably $\mathcal{H}^{N-1}$-rectifiable set. Recalling the definition of normal traces given in Section 2.4, from (61) we deduce that

$$
\left\{\begin{array}{l}
(1-\lambda)\left[\operatorname{Tr}^{i}(\boldsymbol{A}, \Sigma)-\operatorname{Tr}^{e}(\boldsymbol{A}, \Sigma)\right] \leq 0, \\
-\lambda\left[\operatorname{Tr}^{i}(\boldsymbol{A}, \Sigma)-\operatorname{Tr}^{e}(\boldsymbol{A}, \Sigma)\right] \leq 0,
\end{array} \quad \mathcal{H}^{N-1} \text {-a.e. on } \Sigma .\right.
$$

Let us choose an orientation for the countably $\mathcal{H}^{N-1}$-rectifiable set $\Sigma^{+}:=\Theta^{r} \cap \Omega_{\boldsymbol{A}}^{+}$. Since $\Sigma^{+} \subset \Omega_{\boldsymbol{A}}$ and $\psi_{\boldsymbol{A}}(x)=1$ for $|\operatorname{div} \boldsymbol{A}|$-a.e. $x \in \Sigma^{+}$, from (14) we have that

$$
\operatorname{div} \boldsymbol{A}\left\llcorner\Sigma^{+}=\left[\operatorname{Tr}^{i}\left(\boldsymbol{A}, \Sigma^{+}\right)-\operatorname{Tr}^{e}\left(\boldsymbol{A}, \Sigma^{+}\right)\right] \mathcal{H}^{N-1}\left\llcorner\Sigma^{+}>0 .\right.\right.
$$


Hence, from the first inequality in (62), we deduce that $\lambda=1 \mathcal{H}^{N-1}$-a.e. on $\Sigma^{+}$. A similar argument, using $\Sigma^{-}:=\Theta_{\boldsymbol{A}}^{r} \cap \Omega_{\boldsymbol{A}}^{-}$, shows that $\lambda=0 \mathcal{H}^{N-1}$-a.e. on $\Sigma^{-}$.

Corollary 7.7. Let $\boldsymbol{A} \in \mathcal{D} \mathcal{M}^{\infty}(\Omega)$ and let $\lambda: \Omega \rightarrow[0,1]$ be a Borel function. Then the continuity property

$$
\left\langle(\boldsymbol{A}, D u)_{\lambda}, \varphi\right\rangle=\lim _{n}\left\langle\left(\boldsymbol{A}, D u_{n}\right)_{\lambda}, \varphi\right\rangle \quad \forall \varphi \in C_{c}^{\infty}(\Omega),
$$

holds for every $u_{n}, u \in B V(\Omega)$ satisfying (a) and (b) in Theorem 7.6 if and only if $\mathcal{H}^{N-1}\left(\Theta_{\boldsymbol{A}}^{r}\right)=0$.

Proof. We have that the stated property holds if and only if both (55) and (56) hold. From Theorem 7.6, these inequalities hold (for every $\left(u_{n}\right), u$ ) if and only if $\lambda \in \Lambda_{\text {lsc }} \cap \Lambda_{\text {usc }}$. Finally, from the very definition of $\Lambda_{\mathrm{lsc}}$ and $\Lambda_{\text {usc }}$, we have that $\Lambda_{\mathrm{lsc}} \cap \Lambda_{\text {usc }} \neq \emptyset$ if and only if $\mathcal{H}^{N-1}\left(\Theta_{A}^{r}\right)=0$.

Remark 7.8. The assumption $\mathcal{H}^{N-1}\left(\Theta_{\boldsymbol{A}}^{r}\right)=0$ is trivially satisfied if $\operatorname{div}^{j} \boldsymbol{A}=0$, e.g. if $\operatorname{div} \boldsymbol{A} \in L^{1}(\Omega)$.

Example 7.9. In view of Corollary 7.7 we have that, in general, the continuity property (63) does not hold with respect to the strict convergence in $B V$. Specifically, let $\Omega=(-2,2) \subset$ $\mathbb{R}$ and consider $\boldsymbol{A}:=\chi_{(-1,1)}$, so that $\operatorname{div} \boldsymbol{A}=\delta_{-1}-\delta_{1}$, and $\Theta_{\boldsymbol{A}}^{r}=\Theta_{\boldsymbol{A}}=\{-1,+1\}$ is not empty. Let $\lambda: \Omega \rightarrow[0,1]$ be any Borel function. Let $u_{n}(x):=\max \{\min \{n+1-n|x|, 1\}, 0\}$. It is readily seen that $\left(u_{n}\right)$ strictly converges to $u:=\chi_{[-1,1]}$, so that $\left(-u_{n}\right)$ strictly converges to $-u$, and $\left\langle\left(\boldsymbol{A}, D u_{n}\right)_{\lambda}, \varphi\right\rangle=0$ for every $n$. On the other hand, choosing $\varphi$ such that $\varphi(-1)=0$ and $\varphi(1)=1$, one has

$$
\begin{gathered}
\left\langle(\boldsymbol{A}, D u)_{\lambda}, \varphi\right\rangle=[1-\lambda(-1)] \varphi(-1)+[\lambda(1)-1] \varphi(1)=\lambda(1)-1, \\
\left\langle(\boldsymbol{A}, D(-u))_{\lambda}, \varphi\right\rangle=-\lambda(-1) \varphi(-1)+\lambda(1) \varphi(1)=\lambda(1),
\end{gathered}
$$

and at least one of the right-hand sides must be different from 0 .

Example 7.10. We remark that, in general, (55) does not hold if assumption (a) is replaced by the weak* convergence in $B V$. Specifically, let us consider $\Omega=(-2,2) \subset \mathbb{R}, \boldsymbol{A}:=\chi_{(0,1)}$ and $u_{n}(x):=\max \{1-n|x|, 0\}$. Since $\left(u_{n}\right)$ converges to $u=0$ in $L^{1}(\Omega)$ and $\left|D u_{n}\right|(\Omega)=2$ for every $n$, then $\left(u_{n}\right)$ converges weakly* to $u$ in $B V(\Omega)$. (see [3, Proposition 3.13]): If $\varphi \in C_{c}^{\infty}(\Omega)$ is strictly positive in 0 , one has

$$
\begin{aligned}
\liminf _{n}\left\langle\left(\boldsymbol{A}, D u_{n}\right)_{\lambda}, \varphi\right\rangle & =\liminf _{n}\left(-u_{n}(0) \varphi(0)+u_{n}(1) \varphi(1)-\int_{0}^{1} u_{n} \varphi^{\prime}\right) \\
& =-\varphi(0)<0=\left\langle(\boldsymbol{A}, D u)_{\lambda}, \varphi\right\rangle,
\end{aligned}
$$

so that (55) does not hold.

Acknowledgments. The authors would like to thank Giovanni E. Comi for some useful remarks on a preliminary version of the manuscript. A.M. and V.D.C. have been partially supported by the Gruppo Nazionale per l'Analisi Matematica, la Probabilità e le loro Applicazioni (GNAMPA) of the Istituto Nazionale di Alta Matematica (INdAM). G.C. and A.M. have been partially supported by Sapienza - Ateneo 2017 Project "Differential Models in Mathematical Physics". 


\section{REFERENCES}

[1] L. Ambrosio, G. Crippa, and S. Maniglia, Traces and fine properties of a BD class of vector fields and applications, Ann. Fac. Sci. Toulouse Math. (6) 14 (2005), no. 4, 527-561. MR2188582

[2] L. Ambrosio, C. De Lellis, and J. Malý, On the chain rule for the divergence of BV-like vector fields: applications, partial results, open problems, Perspectives in nonlinear partial differential equations, 2007, pp. 31-67. MR2373724

[3] L. Ambrosio, N. Fusco, and D. Pallara, Functions of bounded variation and free discontinuity problems, Oxford Mathematical Monographs, The Clarendon Press Oxford University Press, New York, 2000. MR1857292 (2003a:49002)

[4] F. Andreu, C. Ballester, V. Caselles, and J.M. Mazón, The Dirichlet problem for the total variation flow, J. Funct. Anal. 180 (2001), no. 2, 347-403. MR1814993

[5] F. Andreu-Vaillo, V. Caselles, and J.M. Mazón, Parabolic quasilinear equations minimizing linear growth functionals, Progress in Mathematics, vol. 223, Birkhäuser Verlag, Basel, 2004. MR2033382

[6] G. Anzellotti, Pairings between measures and bounded functions and compensated compactness, Ann. Mat. Pura Appl. (4) 135 (1983), 293-318 (1984). MR750538

[7] M. Carriero, G. Dal Maso, A. Leaci, and E. Pascali, Relaxation of the nonparametric plateau problem with an obstacle, J. Math. Pures Appl. (9) 67 (1988), no. 4, 359-396. MR978576

[8] V. Caselles, On the entropy conditions for some flux limited diffusion equations, J. Differential Equations 250 (2011), no. 8, 3311-3348. MR2772392

[9] G.-Q. Chen, G.E. Comi, and M. Torres, Cauchy fluxes and Gauss-Green formulas for divergencemeasure fields over general open sets, Arch. Rational Mech. Anal. (2018). DOI 10.1007/s00205-01801355-4.

[10] G.-Q. Chen and H. Frid, Divergence-measure fields and hyperbolic conservation laws, Arch. Ration. Mech. Anal. 147 (1999), no. 2, 89-118. MR1702637

[11] _ Extended divergence-measure fields and the Euler equations for gas dynamics, Comm. Math. Phys. 236 (2003), no. 2, 251-280. MR1981992

[12] G.-Q. Chen and M. Torres, Divergence-measure fields, sets of finite perimeter, and conservation laws, Arch. Ration. Mech. Anal. 175 (2005), no. 2, 245-267. MR2118477

[13] On the structure of solutions of nonlinear hyperbolic systems of conservation laws, Commun. Pure Appl. Anal. 10 (2011), no. 4, 1011-1036. MR2787432 (2012c:35263)

[14] G.-Q. Chen, M. Torres, and W.P. Ziemer, Gauss-Green theorem for weakly differentiable vector fields, sets of finite perimeter, and balance laws, Comm. Pure Appl. Math. 62 (2009), no. 2, 242-304. MR2468610

[15] G.E. Comi and V. Magnani, On the Gauss-Green theorem in stratified groups (2018). arxiv:1806.04011.

[16] G.E. Comi and K.R. Payne, On locally essentially bounded divergence measure fields and sets of locally finite perimeter, Adv. Calc. Var. (2017). DOI 10.1515/acv-2017-0001.

[17] G. Crasta and V. De Cicco, An extension of the pairing theory between divergence-measure fields and BV functions, J. Funct. Anal. 276 (2019), no. 8, 2605-2635. MR3926127

[18] - On the chain rule formulas for divergences and applications to conservation laws, Nonlinear Anal. 153 (2017), 275-293. MR3614672

[19] _ Anzellotti's pairing theory and the Gauss-Green theorem, Adv. Math. 343 (2019), 935-970. MR3892346

[20] V. De Cicco, N. Fusco, and A. Verde, A chain rule formula in BV and application to lower semicontinuity, Calc. Var. Partial Differential Equations 28 (2007), no. 4, 427-447. MR2293980 (2007j:49016)

[21] V. De Cicco, D. Giachetti, F. Oliva, and F. Petitta, Dirichlet problems with 1-laplacian principal part and strong singularities, 2017. Preprint.

[22] V. De Cicco, D. Giachetti, and S. Segura De León, Elliptic problems involving the 1-Laplacian and a singular lower order term, J. Lond. Math. Soc. (2018). DOI 10.1112/jlms.12172.

[23] M. Degiovanni, A. Marzocchi, and A. Musesti, Cauchy fluxes associated with tensor fields having divergence measure, Arch. Ration. Mech. Anal. 147 (1999), no. 3, 197-223. MR1709215

[24] I. Fonseca and G. Leoni, Modern methods in the calculus of variations: $L^{p}$ spaces, Springer Monographs in Mathematics, Springer, New York, 2007. MR2341508

[25] M. Giaquinta, G. Modica, and J. Souček, Cartesian currents in the calculus of variations. I, Ergebnisse der Mathematik und ihrer Grenzgebiete. 3. Folge. A Series of Modern Surveys in Mathematics [Results in Mathematics and Related Areas. 3rd Series. A Series of Modern Surveys in Mathematics], vol. 37, Springer-Verlag, Berlin, 1998. Cartesian currents. MR1645086 (2000b:49001a) 
[26] G. Huisken and T. Ilmanen, The inverse mean curvature flow and the Riemannian Penrose inequality, J. Differential Geom. 59 (2001), no. 3, 353-437. MR1916951

[27] B. Kawohl, On a family of torsional creep problems, J. Reine Angew. Math. 410 (1990), 1-22.

[28] P. Lahti, Strict and pointwise convergence of BV functions in metric spaces, J. Math. Anal. Appl. 455 (2017), 1005-1021.

[29] M. Latorre and S. Segura De León, Existence and comparison results for an elliptic equation involving the 1-Laplacian and $L^{1}$-data, J. Evol. Equ. 18 (2018), no. 1, 1-28. MR3772837

[30] G.P. Leonardi and G. Saracco, Rigidity and trace properties of divergence-measure vector fields, 2017. Preprint.

[31] - The prescribed mean curvature equation in weakly regular domains, NoDEA Nonlinear Differential Equations Appl. 25 (2018), no. 2, Art. 9, 29. MR3767675

[32] J.M. Mazón, The Euler-Lagrange equation for the anisotropic least gradient problem, Nonlinear Anal. Real World Appl. 31 (2016), 452-472. MR3490852

[33] J.M. Mazón, J.D. Rossi, and S. Segura de León, Functions of least gradient and 1-harmonic functions, Indiana Univ. Math. J. 63 (2014), no. 4, 1067-1084. MR3263922

[34] C. Scheven and T. Schmidt, BV supersolutions to equations of 1-Laplace and minimal surface type, J. Differential Equations 261 (2016), no. 3, 1904-1932. MR3501836

[35] _ An Anzellotti type pairing for divergence-measure fields and a notion of weakly super-1harmonic functions, 2017. Preprint.

[36] - On the dual formulation of obstacle problems for the total variation and the area functional, Ann. Inst. H. Poincaré Anal. Non Linéaire 35 (2018), no. 5, 1175-1207. MR3813962

[37] F. Schuricht, A new mathematical foundation for contact interactions in continuum physics, Arch. Ration. Mech. Anal. 184 (2007), no. 3, 495-551. MR2299760

[38] M. Silhavý, Divergence measure fields and Cauchy's stress theorem, Rend. Sem. Mat. Univ. Padova 113 (2005), 15-45. MR2168979

Dipartimento di Matematica “G. Castelnuovo", Sapienza Università di Roma, P.le A. Moro 5 - I-00185 Roma (ITALY)

E-mail address: crasta@mat.uniroma1.it

Dipartimento di Scienze di Base e Applicate per l'Ingegneria, Sapienza Università di Roma, Via A. Scarpa 10 - I-00185 Roma (ItAly)

E-mail address: virginia.decicco@sbai.uniroma1.it

Dipartimento di Matematica "G. Castelnuovo", Sapienza Università di Roma, P.le A. Moro 5 - I-00185 Roma (ITALY)

E-mail address: malusa@mat.uniroma1.it 